Research Article

\title{
Electromechanical Performance Analysis of the Hybrid Piezoelectric-Electromagnetic Energy Harvester under Rotary Magnetic Plucking Excitation
}

\author{
Hongyan Wang $\mathbb{D}^{1},{ }^{1}$ Jiarui $\mathrm{Hu},{ }^{1}$ Gang Sun, ${ }^{1}$ and Liying Zou ${ }^{2}$ \\ ${ }^{1}$ College of Mechatronic Engineering, Qiqihar University, Qiqihar 161006, China \\ ${ }^{2}$ College of Communication and Electronics Engineering, Qiqihar University, Qiqihar 161006, China
}

Correspondence should be addressed to Hongyan Wang; wanghongyan1993@163.com

Received 6 March 2021; Revised 17 July 2021; Accepted 2 August 2021; Published 18 August 2021

Academic Editor: Vasudevan Rajamohan

Copyright (c) 2021 Hongyan Wang et al. This is an open access article distributed under the Creative Commons Attribution License, which permits unrestricted use, distribution, and reproduction in any medium, provided the original work is properly cited.

\begin{abstract}
This paper presents an analysis of the hybrid piezoelectric-electromagnetic energy harvester (P-EMEH) driven by contactless rotary magnetic plucking. A lumped-parameter model of the hybrid P-EMEH is developed, and the model parameters are determined from the finite element analysis (FEA) method. A parametric study is conducted to investigate the effects of driving force parameters, load resistance, and electromechanical coupling strengths (EMCSs) on the maximal displacements and velocities, average power inputs and outputs, and energy efficiencies of the system for indicating the performance of the hybrid P-EMEH. The results show that the hybrid P-EMEH can obtain the improved power inputs by reducing the gyration radii of the rotary magnet and shortening the gaps between the two magnets. The structural vibrations can be strongly suppressed owing to the optimal piezoelectric power outputs, which can lead to the occurrence of valleys' power of the electromagnetic element. At weak coupling, the hybrid P-EMEH can achieve higher power outputs than the single piezoelectric energy harvester (PEH) and the single electromagnetic energy harvester (EMEH). At strong coupling, the use of the PEH is more advantageous for energy harvesting due to wider power bandwidths at high dimensionless frequencies when compared with the hybrid P-EMEH. This work provides a fundamental understanding on the effect of load resistance and EMCSs on the dynamic and electrical characteristics of the magnetically plucked hybrid P-EMEH.
\end{abstract}

\section{Introduction}

With the rapid development of the self-sustained sensor networks and wireless communication technology, scavenging energy from the ambient environment to generate sustainable electricity for the low-power electronic devices and the wireless sensor nodes has received extensive attention. Various types of energy sources, such as light, heat, radio frequency, and mechanical motions, can be extracted from the ambient environment [1]. Among these, mechanical vibrations have received the most attention in the past few years because of their abundance in the ambient environment [2]. The rotational vibration is often visible in the ambient environment, ranging from fluid flow [3] and machinery operation [4] to human motion [5]. The rotational vibration energy can be converted into electricity by various transduction mechanisms including piezoelectric [6], electromagnetic [7], electrostatic [8], and triboelectric [9]. Among these, the piezoelectric and electromagnetic energy harvesters have been paid much more attention from researchers due to their advantages of simple configuration and high output performance.

The researchers have proposed various types of rotational energy harvesters for vibration energy harvesting from rotating environments, such as the torsional piezoelectric vibration energy harvesters attached to a rotary oil drilling shaft [10], the rotating piezoelectric vibration energy harvesters based on the gravitational and centrifugal forces' actions [11-13], the mechanically plucked piezoelectric energy harvesters [14], the magnetically plucked 
piezoelectric energy harvesters $[15,16]$, and the rotational electromagnetic energy harvesters based on rotational electromagnetic generator designs $[7,17]$. Among these, the frequency upconversion rotational energy harvesters through magnetic plucking have gained more attention in recent years as they have the advantages of the contactless excitation design and high capability of problem solving in the frequency mismatch between the excitation and the harvester. In terms of the influence of system parameters, the studies on magnetically plucked rotational energy harvesters have mainly focused on excitation frequencies [16, 18], magnet arrangements [16-21], and rectified power estimation [22], while less attention has been paid on the effect of the load resistance and the mechanical coupling strength on the performance of the system, which is significant to design and optimize the magnetically plucked rotational energy harvester.

This work aims to conduct a comprehensive analysis of the effect of the load resistance and the EMCSs on the performance of the hybrid piezoelectric-electromagnetic energy harvester (P-EMEH) driven by the rotary magnetic plucking. The proposed harvester in this study consists of a clamped-clamped piezoelectric beam, three magnets, and a stationary coil. A lumped-parameter model of the hybrid $\mathrm{P}-\mathrm{EMEH}$ is established to analyze the performance of the harvester. The mechanical parameters and the piezoelectric EMCS are determined from the coupled electromechanical FEA. The electromagnetic electromechanical coupling coefficient (EMCC) and the equivalent magnetization of the magnets for the magnetic driving force calculation are determined from the magnetic field finite element analysis. Based on the determined parameters, a parametric study is then performed to analyze the effects of the dimensionless load resistance and dimensionless EMCSs on the maximal displacements and velocities, average power inputs and outputs, and energy conversion efficiencies for indicating the performance of the hybrid P-EMEH. The power performance of the hybrid P-EMEH is also investigated by comparison with the single $\mathrm{PEH}$ and the single EMEH at weak and strong couplings.

\section{Configuration of the Hybrid P-EMEH}

Figures 1(a) and 1(b) show the physical model of a hybrid $\mathrm{P}$-EMEH and its simplified model, respectively. It can be seen from Figure 1(a) that the hybrid P-EMEH consists of a clamped-clamped metal beam with two piezoelectric plates located at two sides of a central magnetic mass on the beam and an external induction coil fixed to a holder. The central magnetic mass includes a lower cuboidal magnet (magnet 2), an upper cylindered magnet (magnet 3), and a middle magnet holder. Magnet 2 is used to match an external rotary cuboidal magnet (magnet 1 ) to generate the magnet driving force. Magnet 3 is used to match a fixed coil to generate the induction current. The hybrid P-EMEH can be simplified as a single-degree-of-freedom mass-spring-damper model with a piezoelectric transducer and an electromagnetic transducer, as shown in Figure 1(b). The parameters $m, k$, and $c$ are the equivalent mass, the equivalent stiffness, and the viscous damping of the harvester, respectively. $C_{p}$ is the capacitance of the two piezoelectric plates in parallel. $L_{c}$ and $R_{c}$ are the internal induction and the internal resistance of the coil, respectively. $\theta_{p}$ and $\theta_{e}$ are the EMCCs related with the piezoelectric and electromagnetic elements, respectively. $R_{1}$ and $R_{2}$ are the load resistance connected to the piezoelectric and electromagnetic elements, respectively. $F_{z}$ and $u$ are the driven force and the vibration displacement of the mass $m$, respectively.

Figures 2(a) and 2(b) show the relative positions among the three magnets and a coil in the three-dimensional spatial direction. From Figure 2(a), it can be seen that magnets 1 and 2 have the sizes of $2 A_{1} \times 2 B_{1} \times 2 C_{1}$ and $2 A_{2} \times 2 B_{2} \times 2 C_{2}$, respectively. Magnet 3 has the radius of $r_{3}$ and the height of $h_{3} . J_{1}, J_{2}$, and $J_{3}$ are the magnetizations of magnets 1,2 , and 3 , respectively. The interaction of magnets 1 and 2 is repulsive due to the opposite direction of the magnetization. The interaction of magnets 2 and 3 is attractive due to the same direction of the magnetization. $d_{x}, d_{y}$, and $d_{z}$ represent the central distances between magnets 1 and 2 in three axes, respectively. $z_{3}$ presents the central distance between magnets 2 and 3 in the $Z$-axis. It can be seen from Figure 2(b) that the fixed coil has the inner radius of $r_{\text {min }}$, the outer radius of $r_{\text {max }}$, and the height of $h_{c}$. When magnet 3 has the vibration displacement of $u$, the distance from the center of magnet 3 to the upper surface of the fixed coil is $z_{c}=z_{c 0}-u$, where $z_{c 0}$ is the initial position of magnet 3 in the $Z_{c}$-axis.

\section{Theoretical Model}

The governing differential equation of the hybrid P-EMEH with the magnetic force excitation can be written as

$$
m \ddot{u}=F_{z}-c \dot{u}-k u-\theta_{p} V_{p}-\theta_{e} I_{e},
$$

where $(\cdot)=\partial / \partial t, V_{p}$ is the voltage across the load resistor $R_{1}$, and $I_{e}$ is the current through the load resistor $R_{2}$. In this research, the magnetic interaction force is induced by the interaction between magnets 1 and 2 in the $z$-direction, which can be written as [23]

$$
F_{z}=\frac{J_{1} \cdot J_{2}}{4 \pi \mu_{0}} \sum_{i=0}^{1} \sum_{j=0}^{1} \sum_{k=0}^{1} \sum_{l=0}^{1} \sum_{p=0}^{1} \sum_{q=0}^{1}(-1)^{i+j+k+l+p+q} \phi_{z},
$$

where $\mu_{0}$ is the vacuum permeability. The parameters $i, j, k, l$, $p$, and $q$ are equal to 0 or 1 according to the specific corner. The parameter $\phi_{z}$ is a function of the magnet dimensions and their gaps in 3 axes, which is given by

$$
\begin{aligned}
\phi_{z}= & -u_{i j} w_{p q} \ln \left(r-u_{i j}\right) \\
& -v_{k l} w_{p q} \ln \left(r-v_{k l}\right) \\
& +u_{i j} v_{k l} \tan ^{-1} \frac{u_{i j} v_{k l}}{r w_{p q}}-r w_{p q},
\end{aligned}
$$

where the lengths $u_{i j}, v_{k l}$, and $w_{p q}$ correspond to the distance between the cube corners and their projections on the axes. $r$ is a function of the parameters of $u_{i j}, v_{k l}$, and $w_{p q}$. These parameters are expressed as [23] 

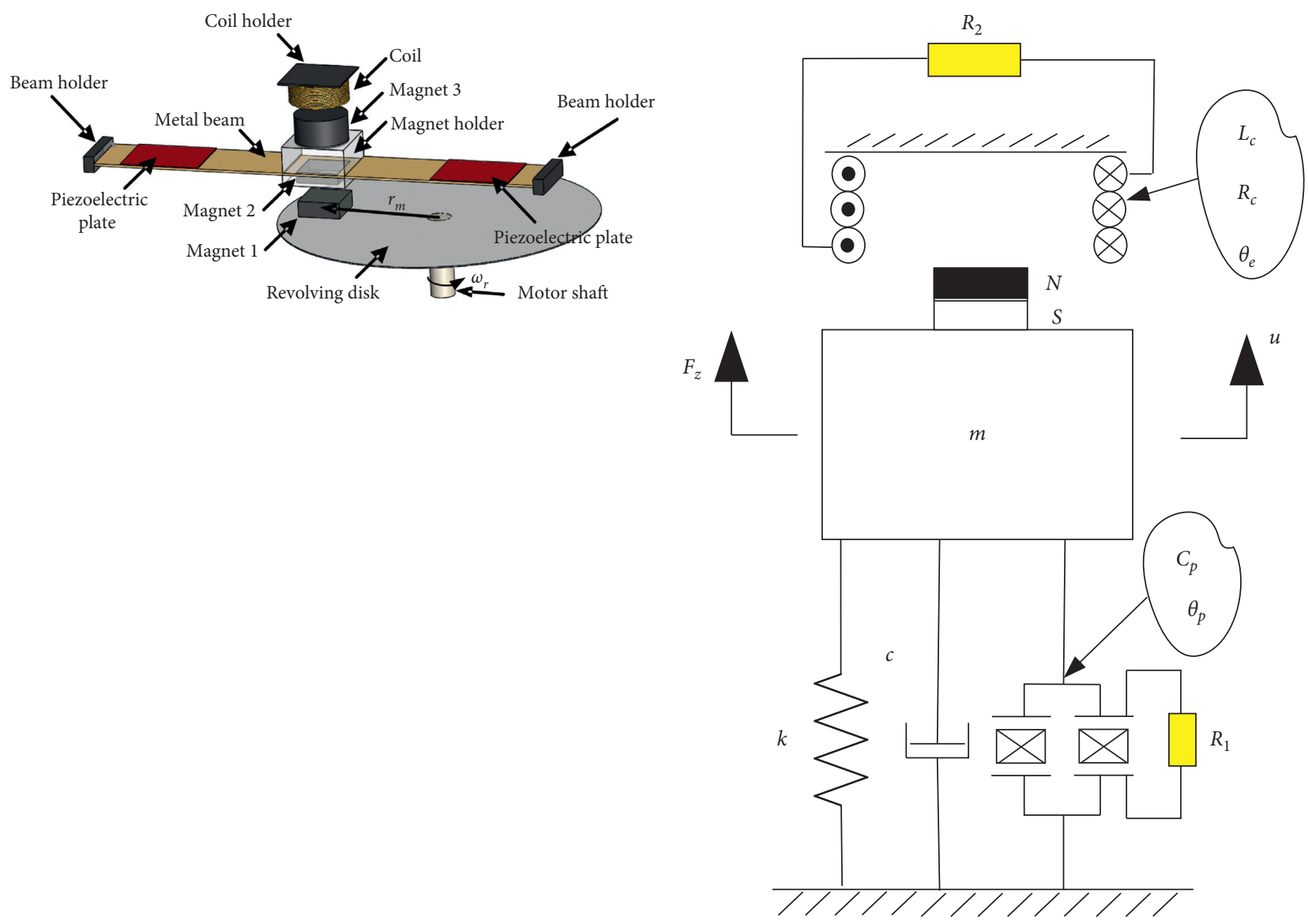

(a)

(b)

Figure 1: (a) Physical model and (b) simplified model of the hybrid P-EMEH.

$$
\begin{aligned}
u_{i j} & =d_{x}+(-1)^{j} A_{2}-(-1)^{i} A_{1}, \\
v_{k l} & =d_{y}+(-1)^{l} B_{2}-(-1)^{k} B_{1}, \\
w_{p q} & =d_{z}+(-1)^{q} C_{2}-(-1)^{p} C_{1}, \\
r & =\left(u_{i j}^{2}+v_{k l}^{2}+w_{p q}^{2}\right)^{1 / 2} .
\end{aligned}
$$

When the revolving disk with magnet 1 rotates with the motor shaft (see Figure 1(a)), the gaps $d_{x}, d_{y}$, and $d_{z}$ between magnets 1 and 2 can be expressed as [16]

$$
\begin{aligned}
& d_{x}=r_{m}+d_{x 0}-r_{m} \cos \left(\omega_{r} t-\varphi_{0}\right), \\
& d_{y}=r_{m} \sin \left(\omega_{r} t-\varphi_{0}\right)+d_{y 0}, \\
& d_{z}=d_{z 0}+u
\end{aligned}
$$

where $\omega_{r}$ is the rotational angular frequency of the motor shaft, $r_{m}$ is the gyration radius of magnet $1, d_{x 0}, d_{y 0}$, and $d_{z 0}$ are the initial gaps between magnets 1 and 2 in the $x_{-}, y_{-}$, and $z$-axes, respectively, and $\phi_{0}$ is the initial angular position of magnet 1 relative to magnet 2 . In this paper, we determine $d_{x 0}=d_{y 0}=\phi_{0}=0$.

The electromagnetic EMCC, $\theta_{e}$, can be obtained by calculating the gradient of the magnetic flux in the coil:

$$
\theta_{e}=-\frac{\mathrm{d} \Phi}{\mathrm{d} u}
$$

where $\Phi$ is the total magnetic flux in the coil, $\Phi=N_{c} \xi \Phi_{a}$, where $\Phi_{a}$ is the magnetic flux per unit cross-sectional area of the coil. $N_{c}$ is the number of turns in the coil. $\xi$ is the fill factor of the coil, $\xi=N_{c} A_{w} / A_{c}$, where $A_{w}$ is the conductive area of a single wire, $A_{w}=\pi d_{w}^{2} / 4$, where $d_{w}$ is the conductive diameter of a single wire. $A_{c}$ is the cross-sectional area of the coil, $A_{c}=\left(r_{\max }-r_{\min }\right) h_{c}$.

The magnetic field at the position of a point of the coil is given by [24]

$$
\begin{aligned}
\vec{B} & =-\frac{\mu_{0}}{4 \pi} \vec{\nabla} \frac{\vec{\sigma} \cdot \vec{s}}{s^{3}} \\
& =\frac{\mu_{0}}{4 \pi s^{5}}\left[3(\vec{\sigma} \cdot \vec{s}) \vec{s}-r^{2} \vec{\sigma}\right],
\end{aligned}
$$

where $\vec{\nabla}$ is a vector gradient, $\vec{\sigma}$ is the magnetic dipole moment of magnet $3, \vec{s}$ is a vector from the magnetic core to a point of the coil, and $s$ is its magnitude. According to Figure 2(b), $\vec{s}=r \widehat{e}_{r}+\left(z_{20}-u-z_{1}\right) \widehat{e}_{z}$, where $r$ is the radius of the wire loop and $\widehat{e}_{r}$ and $\widehat{e}_{z}$ are the unit vector along the $x_{c^{-}}$ and $z_{c}$-axis, respectively. Substituting the vector $\vec{s}=r \widehat{e}_{r}+$ 
$\left(z_{20}-u-z_{1}\right) \widehat{e}_{z}$ into equation (7) and simplifying this equation, the magnetic field $\vec{B}$ can be rewritten as

$$
\vec{B}=\frac{\mu_{0} \sigma}{4 \pi}\left(\frac{3 r\left(z_{c}-z\right) \widehat{e}_{r}-\left(r^{2}-2\left(z_{c}-z\right)^{2}\right) \widehat{e}_{z}}{\left(r^{2}+\left(z_{c}-z\right)^{2}\right)^{5 / 2}}\right),
$$

where $\sigma$ is the magnitude of the magnetic dipole moment of magnet 3. Dot multiplication of vectors $\vec{B}$ and $\widehat{e}_{z}$ and then taking integration of the surface of a circle enclosed by a wire loop, the normal component of the magnetic flux through a wire loop with the radius $r$ can be calculated as

$$
\begin{aligned}
\Phi_{z} & =\int_{0}^{2 \pi} \int_{0}^{r} \vec{B} \widehat{e}_{z}(r \mathrm{~d} r \mathrm{~d} \theta) \\
& =\frac{\mu_{0} \sigma}{2} \frac{r^{2}}{\left(r^{2}+\left(z_{c}-z\right)^{2}\right)^{3 / 2}} .
\end{aligned}
$$

Integrating $\Phi_{z}$ given in equation (9) over the whole cross section of the coil and dividing by the area $A_{c}$, the average magnetic flux through the cross section of the coil can be written as

$$
\begin{aligned}
\Phi_{a} & =\frac{1}{A_{c}} \int_{0}^{h_{c}} \int_{r_{\min }}^{r_{\max }} \Phi_{z} \mathrm{~d} r \mathrm{~d} z \\
& =\frac{1}{A_{c}} \int_{0}^{h_{c}} \int_{r_{\min }}^{r_{\max }} \frac{\mu_{0} \sigma}{2} \frac{r^{2}}{\left(r^{2}+\left(z_{c}-z\right)^{2}\right)^{3 / 2}} \mathrm{~d} r \mathrm{~d} z .
\end{aligned}
$$

Since $z_{c}=z_{c 0}-u$ and $z_{c}$ is not relative to $z$, returning to equation (6), we can rewrite the electromagnetic EMCC as

$$
\begin{aligned}
\theta_{e} & =-N_{c} \xi \frac{\mathrm{d} \Phi_{a}}{\mathrm{~d} u} \\
& =N_{c} \xi \frac{\mathrm{d} \Phi_{a}}{\mathrm{~d}\left(z_{c}-z\right)} .
\end{aligned}
$$

Substituting the expression of $\Phi_{a}$ in equation (10) into equation (11) and then taking the derivative with respect to $z_{c}-z$, the electromagnetic EMCC, $\theta_{e}$, can be written by [25]

$$
\theta_{e}=\frac{N_{c} \xi \mu_{0} \sigma}{2 A_{c}} \sum_{i, j=1}^{2}(-1)^{i+j}\left(\ln \left(r_{c i}+z_{i j}\right)-\frac{r_{c i}}{z_{i j}}\right),
$$

where $z_{i j}^{2}=r_{c i}^{2}+\left(z_{j}-u\right)^{2}$, where $r_{c 1}=r_{\min }, \quad r_{c 2}=r_{\max }$, $z_{1}=z_{c 0}$, and $z_{2}=z_{c 0}-h_{c}$.

Considering the equivalent electrical model of the electromagnetic transducer [26], the circuit governing equation for electromagnetic transduction of the system can be written as

$$
\theta_{e} \dot{u}=L_{c} \dot{I}_{e}+I_{e}\left(R_{c}+R_{2}\right) .
$$

Neglecting the inductance of the coil, which is commonly accepted for low frequencies [27, 28], the following equation can be derived from equations (1) and (13):

$$
m \ddot{u}=F_{z}-\left(c+\frac{\theta_{e}}{R_{c}+R_{2}}\right) \dot{u}-k u-\theta_{p} V_{p} .
$$

Considering the equivalent electrical model of the piezoelectric transducer [26], the circuit governing equation for piezoelectric transduction of the system can be written as

$$
\theta_{p} \dot{u}=C_{p} \dot{V}_{p}+\frac{V_{p}}{R_{1}} .
$$

Defining the state-space vector

$$
\left\{\begin{array}{lll}
x_{1} & x_{2} & x_{3}
\end{array}\right\}^{T}=\left\{\begin{array}{lll}
u & \dot{u} & V_{p}
\end{array}\right\}^{T},
$$

we can write the governing equations of the hybrid P-EMEH from equations (14) and (15) in the state-space form as

$$
\left\{\begin{array}{c}
\dot{x}_{1} \\
\dot{x}_{2} \\
\dot{x}_{3}
\end{array}\right\}=\left\{\begin{array}{c}
x_{2} \\
\frac{F_{z}}{m}-\left(2 \zeta+\frac{k_{e}^{2}}{1+r_{2}}\right) \omega_{n} x_{2}-\omega_{n}^{2} x_{1}-\omega_{n} k_{p} x_{3} \sqrt{\frac{C_{p}}{m}} \\
\omega_{n} k_{p} x_{2} \sqrt{\frac{m}{C_{p}}}-\frac{\omega_{n}}{r_{1}} x_{3}
\end{array}\right\},
$$

where $\quad \omega_{n}=\sqrt{k / m}, \quad \zeta=(c / 2 \sqrt{k m}), \quad k_{p}^{2}=\left(\theta_{p}^{2} / k C_{p}\right)$, $k_{e}^{2}=\left(\theta_{e}^{2} / m \omega_{n} R_{c}\right), r_{1}=R_{1} C_{p} \omega_{n}$, and $r_{2}=\left(R_{2} / R_{c}\right)$.

The average electromagnetic coupling strength $\bar{k}_{e}^{2}$ in a rotational cycle is

$$
\bar{k}_{e}^{2}=\frac{\omega_{r}}{2 \pi} \int_{t_{0}}^{t_{0}+\left(2 \pi / \omega_{r}\right)} k_{e}^{2}(u(t)) \mathrm{d} t,
$$

where $\omega_{r}$ is the rotational angular frequency of the driving magnet and $t_{0}$ is the initial time for a rotational cycle.

The average power input of the system in a rotational cycle is

$$
\bar{P}_{\text {in }}=\frac{\omega_{r}}{2 \pi} \int_{t_{0}}^{t_{0}+\left(2 \pi / \omega_{r}\right)} F_{z}(t) \cdot \dot{u}(t) \mathrm{d} t
$$

The average power outputs on the resistors $R_{1}$ and $R_{2}$ in a rotational cycle are

$$
\begin{aligned}
\bar{P}_{R 1} & =\frac{\omega_{r}}{2 \pi R_{1}} \int_{t_{0}}^{t_{0}+\left(2 \pi / \omega_{r}\right)} V_{p}^{2}(t) \mathrm{d} t \\
& =\frac{\omega_{r} C_{p} \omega_{n}}{2 \pi r_{1}} \int_{t_{0}}^{t_{0}+\left(2 \pi / \omega_{r}\right)} V_{p}^{2}(t) \mathrm{d} t, \\
\bar{P}_{R 2} & =\frac{\omega_{r} R_{2}}{2 \pi} \int_{t_{0}}^{t_{0}+(2 \pi / \omega)} I_{e}^{2}(t) \mathrm{d} t \\
& =\frac{k_{e}^{2} m \omega_{n} \omega_{r}}{2 \pi\left(1+r_{2}\right)} \int_{t_{0}}^{t_{0}+(2 \pi / \omega)} \dot{u}^{2}(t) \mathrm{d} t .
\end{aligned}
$$

The total average power output of the hybrid energy harvesting system is

$$
\bar{P}_{\text {total }}=\bar{P}_{R 1}+\bar{P}_{R 2} \text {. }
$$

The efficiency of the system is 


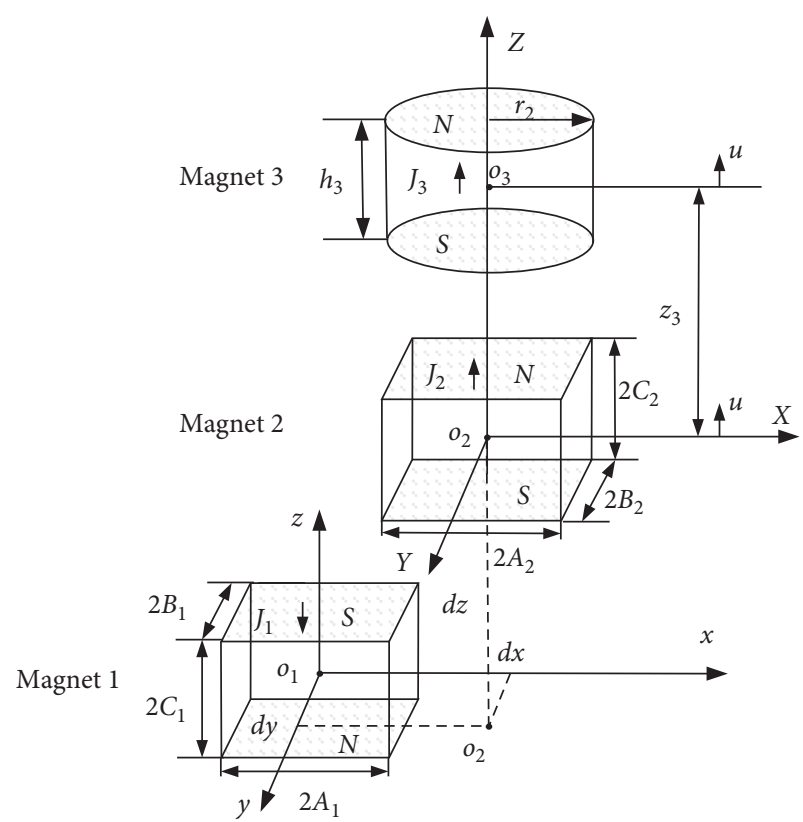

(a)

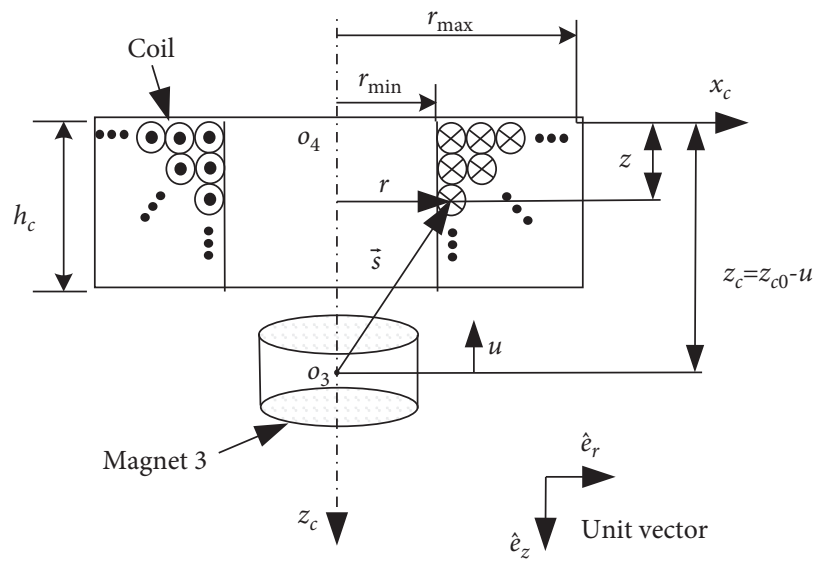

(b)

Figure 2: Relative positions among magnets 1, 2, and 3 and the fixed coil. (a) Magnets 1, 2, and 3. (b) Magnet 3 and the coil.

$$
\eta=\frac{\bar{P}_{\text {total }}}{\bar{P}_{\text {in }}} .
$$

When the coil circuit is open $\left(r_{2}=0\right.$ and $\left.\theta_{e}=0\right)$, equation (17) is reduced to

$$
\left\{\begin{array}{c}
\dot{x}_{1} \\
\dot{x}_{2} \\
\dot{x}_{3}
\end{array}\right\}=\left\{\begin{array}{c}
x_{2} \\
\frac{F_{z}}{m}-2 \zeta \omega_{n} x_{2}-\omega_{n}^{2} x_{1}-\omega_{n} k_{p} x_{3} \sqrt{\frac{C_{p}}{m}} \\
\omega_{n} k_{p} x_{2} \sqrt{\frac{m}{C_{p}}}-\frac{\omega_{n}}{r_{1}} x_{3}
\end{array}\right\},
$$

which is the governing equation of a single $\mathrm{PEH}$ in the statespace form.

When the surface of the piezoelectric element is electrically shorted $\left(r_{1} \longrightarrow \infty\right.$ and $\left.\theta_{p}=0\right)$, equation (17) is reduced to

$$
\left\{\begin{array}{c}
\dot{x}_{1} \\
\dot{x}_{2}
\end{array}\right\}=\left\{\begin{array}{c}
x_{2} \\
\frac{F_{z}}{m}-\left(2 \zeta+\frac{k_{e}^{2}}{1+r_{2}}\right) \omega_{n} x_{2}-\omega_{n}^{2} x_{1}
\end{array}\right\},
$$

which is the governing equation of a single EMEH in the state-space form.

\section{System Parameter Determination}

Based on the material and geometric parameters of the piezoelectric clamped-clamped beam listed in Table 1 and the parameters of magnets and the coil listed in Table 2, in this section, the model parameters of the hybrid P-EMEH, including $\omega_{n}, m, k_{p}^{2}, J_{1}, J_{2}$, and $\sigma$, are determined based on the results from finite element physical field analysis.

The theoretical parameters $\omega_{n}, m$, and $k_{p}^{2}$ are determined from the coupled electromechanical FEA. Figure 3(a) shows the FE model of the clamped-clamped piezoelectric beam. When the circuit is shorted, the node voltage on the upper and lower surface of the two piezoelectric plates is coupled together and set to zero. When the circuit is open, the node voltage on the upper and lower surface of the two piezoelectric plates is coupled separately, and the voltage of the lower surface is set to zero. In FE modal analysis, the shortcircuit and open-circuit resonance frequencies are obtained as $f_{\mathrm{sc}}=122.15 \mathrm{~Hz}$ and $f_{\mathrm{oc}}=122.76 \mathrm{~Hz}$, respectively. According to the equation $k_{p}^{2}=\left(\omega_{\mathrm{oc}}{ }^{2} / \omega_{\mathrm{sc}}{ }^{2}\right)-1[29,30]$, the piezoelectric EMCS can be calculated as $k_{p}^{2}=0.01$, where $\omega_{\mathrm{sc}}$ and $\omega_{\text {oc }}$ are the short-circuit and open-circuit resonant angular frequencies, respectively. The parameters $\omega_{\mathrm{sc}}=2 \pi f_{\mathrm{sc}}$ and $\omega_{\mathrm{oc}}=2 \pi f_{\mathrm{oc}}$. In FE harmonic response analysis, based on the electrical short-circuit condition of the piezoelectric plate and the assumed damping ratio $\zeta=0.02$, a force of $F=1 \mathrm{~N}$ is applied to the center of the magnetic mass in the $z$-direction. Figure 3(b) shows the displacement response amplitudes, $U_{z}$, of the magnetic mass versus the dimensionless rotational frequency $\gamma$. The parameter $\gamma=\omega / \omega_{n}$, where $\omega$ and $\omega_{n}$ are the 
TABLE 1: Material and geometric parameters of the piezoelectric clamped-clamped beam.

\begin{tabular}{lc}
\hline Parameter & Value \\
\hline Clamped length of the metal beam $(\mathrm{mm})$ & 85 \\
Thickness of the metal beam $(\mathrm{mm})$ & 0.4 \\
Length of single piezoelectric plate, $L_{p}(\mathrm{~mm})$ & 15 \\
Thickness of single piezoelectric plate, $h_{p}(\mathrm{~mm})$ & 0.2 \\
Width of the metal beam and piezoelectric plate, $b(\mathrm{~mm})$ & 12 \\
Length of the magnet holder $(\mathrm{mm})$ & 12 \\
Width of the magnet holder $(\mathrm{mm})$ & 12 \\
Central mass of the beam (including the magnet holder and magnets 2 and 3$)(\mathrm{kg})$ & 0.005742 \\
Density of the metal beam $\left(\mathrm{kg} / \mathrm{m}^{3}\right)$ & 8920 \\
Density of the piezoelectric plate $\left(\mathrm{kg} / \mathrm{m}^{3}\right)$ & 7500 \\
Elastic modulus of the metal beam $(\mathrm{GPa})$ & 113 \\
Poisson's ratio of the metal beam & 0.35 \\
Vacuum permittivity of the piezoelectric plate, $e_{0}\left(10^{-12} \mathrm{~F} / \mathrm{m}\right)$ & 8.85 \\
Relative permittivity of the piezoelectric plate, $\varepsilon_{11}^{S} / \varepsilon_{0}, \varepsilon_{33}^{S} / \varepsilon_{0}$ & 1700,1470 \\
Piezoelectric constants, $d_{31}, d_{33}$, and $d_{15}\left(10^{-12} \mathrm{C} / \mathrm{N}\right)$ & $-274,593,741$ \\
Piezoelectric compliance constants, $\varepsilon_{11}^{E}, \varepsilon_{12}^{E}, \varepsilon_{13}^{E}, \varepsilon_{33}^{E}, \varepsilon_{44}^{E}$, and $\varepsilon_{66}^{E}\left(10^{-12} \mathrm{~m}^{2} / \mathrm{N}\right)$ & $16.5,-4.78,-8.45,20.7,43.5,42.6$ \\
\hline
\end{tabular}

TABle 2: Parameters of magnets and the coil.

\begin{tabular}{lc}
\hline Parameter & Value \\
\hline Size of magnet $1, A_{1} \times B_{1} \times C_{1}\left(\mathrm{~mm}^{3}\right)$ & $4 \times 4 \times 1.5$ \\
Size of magnet $2, A_{2} \times B_{2} \times C_{2}\left(\mathrm{~mm}^{3}\right)$ & $4 \times 4 \times 1$ \\
Radius of magnet $3, r_{3}(\mathrm{~mm})$ & 5 \\
Height of magnet $3, h_{3}(\mathrm{~mm})$ & 5 \\
Central distance between magnet 2 and magnet 3 in the $z$-axis, $z_{3}(\mathrm{~mm})$ & 8.5 \\
Residual flux density, $B_{r}(\mathrm{~T})$ & 1.141 \\
Coercive force, $H_{c}(\mathrm{~A} / \mathrm{m})$ & -908775 \\
Permeability of vacuum, $\mu_{0}\left(\mathrm{~N} / \mathrm{A}^{2}\right)$ & $4 \pi \times 10^{-7}$ \\
Height of the coil, $h_{c}(\mathrm{~mm})$ & 5 \\
Inner radius of the coil, $r_{\min }(\mathrm{mm})$ & 6 \\
Outer radius of the coil, $r_{\max }(\mathrm{mm})$ & 9 \\
Turns of the coil, $N_{c}$ & 1000 \\
Conductive diameter of the wire, $d_{w}(\mathrm{~mm})$ & 0.1 \\
Diameter of the wire $(\mathrm{mm})$ & 0.123 \\
Internal resistance $(\Omega)$ & 90 \\
\hline
\end{tabular}

excitation angular frequency and the natural angular frequency, respectively. The parameter $\omega_{n}=\omega_{\text {sc }}$. It can be seen from Figure 3(b) that $U_{z}=5.697 \mathrm{~mm}$ at $\gamma=1$. The equivalent stiffness of the beam can be calculated as $k=4388 \mathrm{~N} / \mathrm{m}$ according to the equation of the magnification factor given in $[31]$.

$$
\frac{U_{z}}{F / k}=\frac{1}{\sqrt{\left(1-\gamma^{2}\right)^{2}+(2 \zeta \gamma)^{2}}}
$$

The equivalent mass of the beam can be calculated as $m=0.0074 \mathrm{~kg}$ according to the equation $m=k / \omega_{n}{ }^{2}$. Since the two piezoelectric plates on the metal beam are electrically connected in parallel, the total capacitance of the piezoelectric element is $C_{p}=2 \varepsilon_{33}^{S} \varepsilon_{0} L_{p} b / h_{p}=23.4 \mathrm{nF}$.

The model parameters $J_{1}$ and $J_{2}$ and $\sigma$ are determined by the FE magnetic field analysis. Note that the magnetic parameters $B_{r}=1.141 \mathrm{~T}$ and $H_{c}=-908775 \mathrm{~A} / \mathrm{m}$ given in Table 2 are provided for FE magnetic field analysis. The magnetic driving force obtained from FEA is used to determine the magnetizations $J_{1}$ and $J_{2}$ used in equation (2). The magnetic flux density obtained from FEA is used to determine the parameter $\sigma$ used in equation (12). Figures 4(a) and 4(b) show the magnetic flux density distribution in the $X O Z$ plane with the magnetic vector plots for the two-magnet and the three-magnet configurations, respectively. It can be seen from Figures 4(a) and 4(b) that, after magnet 3 is introduced to the original magnetic field induced by magnets 1 and 2, the magnetic flux density around magnets 1 and 2 is changed. It implied that magnets 1,2 , and 3 can interact with each other. In this paper, we consider the interaction force between magnets 2 and 3 as the internal force, and thus, equation (2) can be used to calculate the magnetic driving force in the field of the three-magnet configuration. Figures 4(c) and 4(d) show the theoretical and FE magnetic driving force $F_{z}$ versus the gaps $d_{z}$ and $d_{y}$, respectively. It can be seen from Figures $4(\mathrm{c})$ and $4(\mathrm{~d})$ that the theoretical magnetic driving force with the parameters $J_{1}=-J_{2}=1.141 \mathrm{~T}$ and $J_{1}=-J_{2}=1.26 \mathrm{~T}$, respectively, shows good agreements with FEA results for the two-magnet (magnets 1 and 2) and three-magnet (magnets 1, 2, and 3) arrangements. In Section 5 , the parameter $J_{1}=-J_{2}=1.26 \mathrm{~T}$ will be used for the 

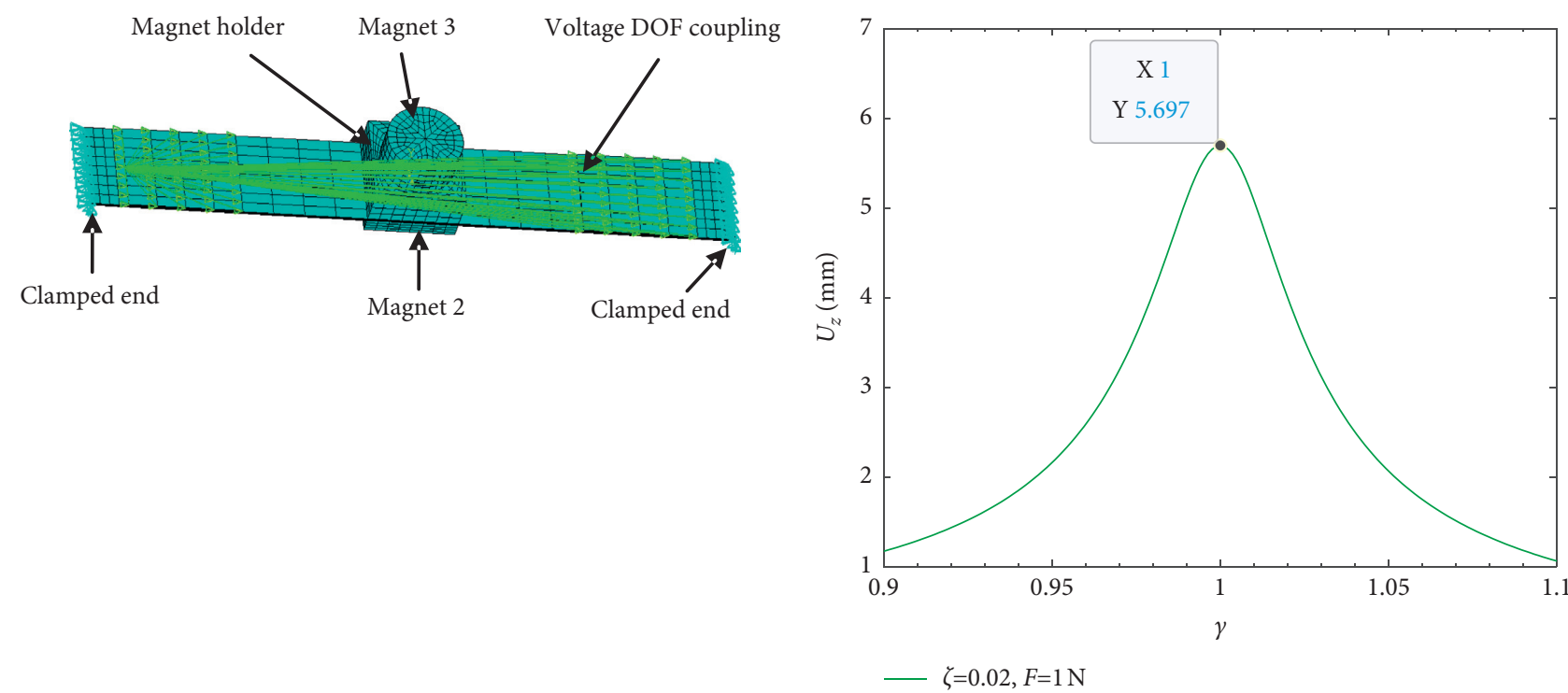

(a)

(b)

FIgURE 3: FE model of the piezoelectric beam and displacement response of magnetic mass under $1 \mathrm{~N}$ applied force. (a) FE model. (b) Displacement versus dimensionless rotational frequency $\gamma$.

theoretical analysis of the dynamics and electrical characteristics of the hybrid P-EMEH.

The magnetic flux $\Phi_{z}$ through an arbitrary wire loop in the normal direction can be obtained from FE magnetic field analysis and also be calculated from the theoretical model (see equation (9)). With a series of selected wire loops, we can determine the average magnetic flux density in the coil, $B_{\text {ave, as }}$

$$
B_{\text {ave }}=\frac{1}{n_{1} n_{2}} \sum_{i=1}^{n_{1}} \sum_{j=1}^{n_{2}} \frac{1}{\pi r_{i}^{2}} \Phi_{z}\left(r_{i}, z_{j}\right),
$$

where $n_{1}$ is the number of wire loops in the $x_{c}$-axis, $n_{2}$ is the number of cross sections of the coil in the $z_{c}$-axis, $\Phi_{z}\left(r_{i}, z_{j}\right)$ is the magnetic flux through a given wire loop, where $r_{i}(i=1$, $\left.2,3, \ldots, n_{1}\right)$ is the radius of an arbitrary wire loop, and $z_{j}$ $\left(j=1,2,3, \ldots, n_{2}\right)$ is the location of the wire loop with respect to the upper surface of the coil along the $z_{c}$-axis (see Figure 2(b)). In FEA, $r_{i}(i=1,2,3$, and 4$)$ is given as $6 \mathrm{~mm}$, $7 \mathrm{~mm}, 8 \mathrm{~mm}$, and $9 \mathrm{~mm}$, respectively. $z_{j}(j=1,2,3$, and 4$)$ is given as $0 \mathrm{~mm}, 1 \mathrm{~mm}, 2 \mathrm{~mm}, 3 \mathrm{~mm}, 4 \mathrm{~mm}$, and $5 \mathrm{~mm}$, respectively. Thus, $n_{1}=4$ and $n_{2}=6$. In the theoretical model, we consider increments of $0.123 \mathrm{~mm}$ from $r_{1}=r_{\min }=6 \mathrm{~mm}$ to $r_{n 1}=r_{\max }=9 \mathrm{~mm}$ in the positive $x_{c}$-axis direction and from $z_{1}=0$ to $z_{n 2}=h_{c}=5 \mathrm{~mm}$ in the positive $z_{2}$-axis direction; thus, $n_{1}=26$ and $n_{2}=41$. Set $z_{20}=8.5 \mathrm{~mm}$ (corresponding to $u=0)$. Figure 5 shows the theoretical average magnetic flux densities as a function of the displacement $u$, which is matched to FEA results. It can be seen from Figure 5 that the theoretical average magnetic flux densities with the parameter $\sigma=0.3428 \mathrm{Am}^{2}$ show good agreements with the results from FEA. The electromagnetic EMCS can be calculated by using the determined value of $\sigma$.

\section{Parametric Study}

In the parametric studies, the differential equations are solved using a Runge-Kutta method. The number of vibration cycles and the number of time steps in one period are given as 20 and 1000, respectively. The constant parameters are given as $m=0.0074 \mathrm{~kg}, w_{n}=2 \pi f_{\mathrm{sc}}=767.5 \mathrm{rad} / \mathrm{s}, \zeta=0.02$, $C_{p}=23.4 \mathrm{nF}$, and $R_{c}=90 \Omega$. The rational radius of magnet 1 and the central distance between magnets 1 and 2 are originally set to be $r_{m(0)}=30 \mathrm{~mm}$ and $d_{z 0(0)}=5 \mathrm{~mm}$, respectively. The dimensionless parameters $\Omega=\omega_{r} / \omega_{n}, \alpha=r_{m} /$ $r_{m(0)}$, and $\beta=d_{z 0} / d_{z 0(0)}$ are used in the parametric analysis. In Section 5.1, the effect of $\Omega, \alpha$, and $\beta$ on the magnetic driving force and the average power inputs are analyzed. In Sections 5.2 and 5.3, the effect of the dimensionless load resistance and the EMCSs on the system performance is analyzed, respectively. In Section 5.4, the power outputs of the hybrid $\mathrm{P}-\mathrm{EMEH}$ at strong and weak couplings are compared with those from the single PEH and the single EMEH.

5.1. Effect of $\Omega, \alpha$, and $\beta$ on Magnetic Driving Force and Average Power Inputs. In this section, the electrical and the electromechanical parameters used for analysis are given as $r_{1}=r_{2}=1$, $k_{p}^{2}=0.01$, and $\sigma=0.3428 \mathrm{Am}^{2}$. Figures 6(a)-6(c) show the time responses of the magnetic driving force and the vibration velocity of the beam for three different $\Omega$, respectively. It can be seen from Figures 6(a)-6(c) that when $\Omega=1$, the harvester works in the resonant mode with a natural vibration period of $8.19 \mathrm{~ms}$, in which the rotational frequency of magnet 1 is synchronized with the beam vibration. When $\Omega=1 / 4$ and $\Omega=1 / 8$, it is observed that although given different excitation periods, $32.75 \mathrm{~ms}$ and $65.5 \mathrm{~ms}$, the harvester vibrates at its natural period of $8.19 \mathrm{~ms}$, which implies that the dominant 


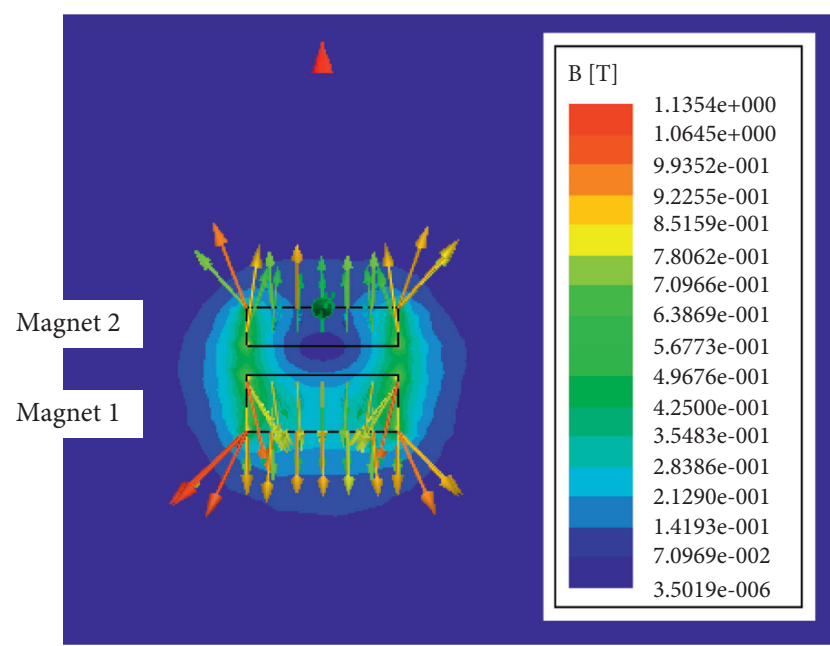

(a)

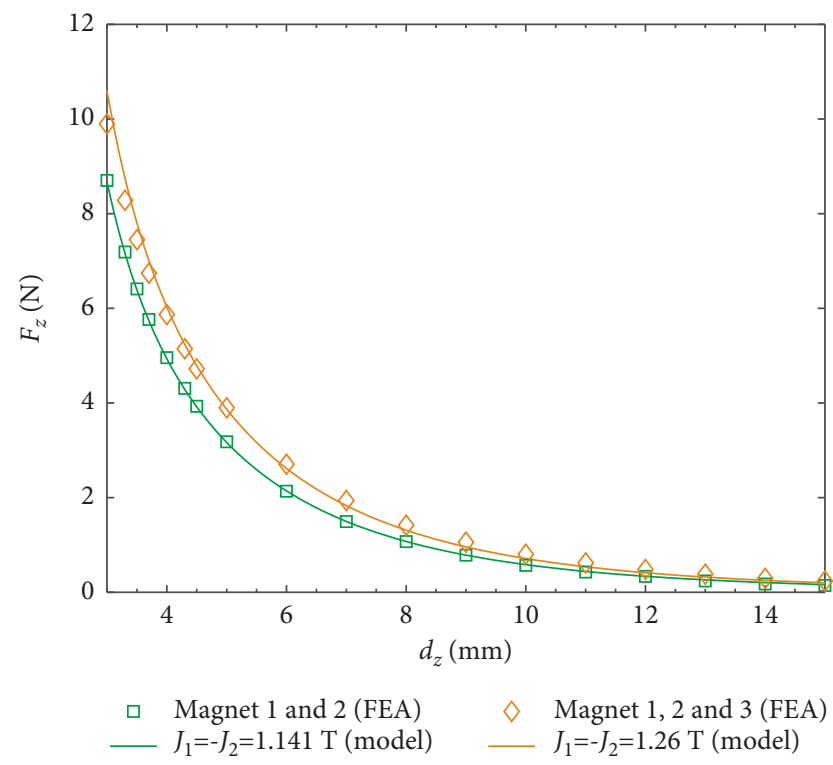

(c)

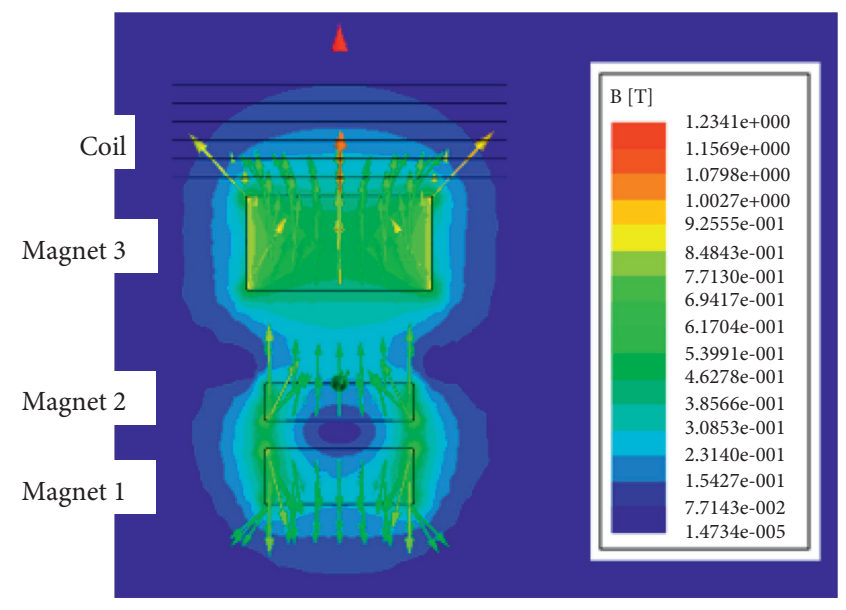

(b)

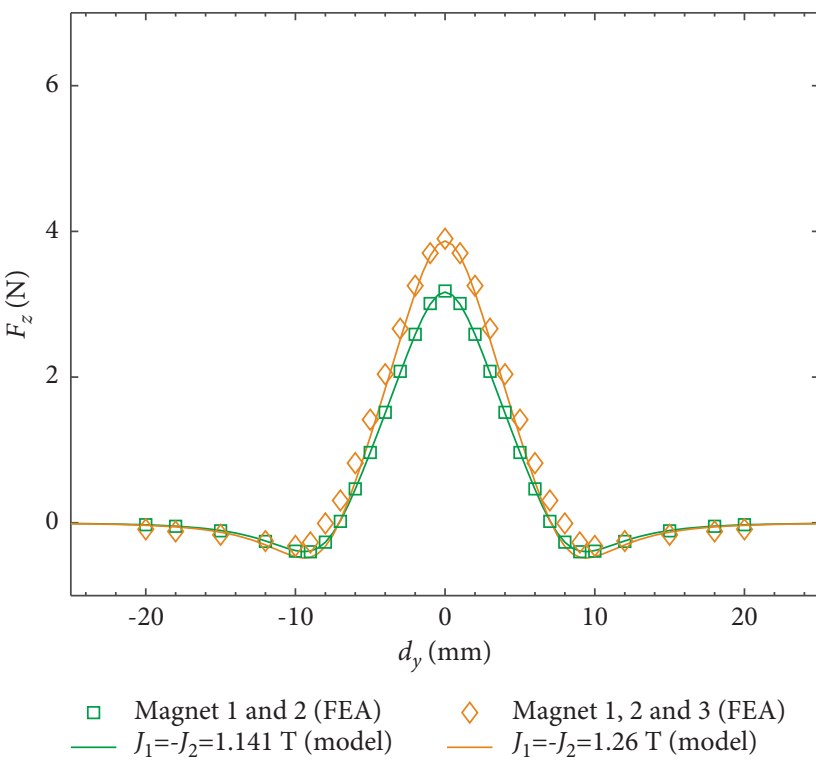

(d)

FIgURe 4: Magnetic flux density distribution in the XOZ plane for the (a) two-magnet configuration and (b) three-magnet configuration from FEA. (c) Magnetic driving force versus $d_{z}$ at $d_{y}=0$. (d) Magnetic driving force versus $d_{y}$ at $d_{z}=0$.

vibration frequency of the harvester is equal to its natural frequency. As we all know, a maximal amplitude of the vibration occurs when a structure vibrates at its natural frequency. It is concluded that a multiple-peak frequency response is available in the nonresonant excitation case. In Figures 6(b) and 6(c), the gradual decay of vibration amplitude between the two excitation cycles indicates that the harvester works in a damped-free-vibration mode after each excitation. It should be noted that the velocity of the beam at off-resonance (see Figures 6(b) and 6(c)) is larger than that of the resonant case (Figure 6(a)). To illustrate the phenomenon, we substitute the parameter $V_{p}$ in equation (15) into (14) and rearrange equation (14) as

$$
\ddot{u}+2 \omega_{n}\left(\zeta+\zeta_{p}+\zeta_{e}\right) \dot{u}+\omega_{n r}^{2} u=\frac{F_{z}}{m},
$$

where $\omega_{n r}$ is the resonant frequency of the system, $\omega_{n r}=\omega_{n} \sqrt{1+\Omega^{2} k_{p}^{2} r_{1}^{2} /\left(1+\Omega^{2} r_{1}^{2}\right)} . \quad \zeta_{p}$ is the piezoelectric damping coefficient, $\zeta_{p}=k_{p}^{2} r_{1} /\left(2\left(1+\Omega^{2} r_{1}^{2}\right)\right)$. $\zeta_{e}$ is the electromagnetic damping coefficient, $\zeta_{e}=k_{e}^{2} /\left(2\left(1+r_{2}\right)\right)$. Considering the average electromagnetic EMCS in a rotational period, the electromagnetic damping coefficients can be written as $\zeta_{e}=\bar{k}_{e}^{2} /\left(2\left(1+r_{2}\right)\right)$. It can be seen from equation (28) that, with a decrease in $\Omega, \zeta_{p}$ increases and $\omega_{n r}$ decreases. Higher $\zeta_{p}$ can lead to a faster decay of the velocity $\dot{u}$ between two excitations. Lower $\omega_{n r}$ means a lower stiffness of the system, which can lead to a higher initial velocity after each excitation. This is the reason why the velocity of the beam at off-resonance (see Figures 6(b) and 6(c)) is larger than that of the resonant case (Figure 6(a)). Figure 6(d) shows the frequency response of the average power inputs of 


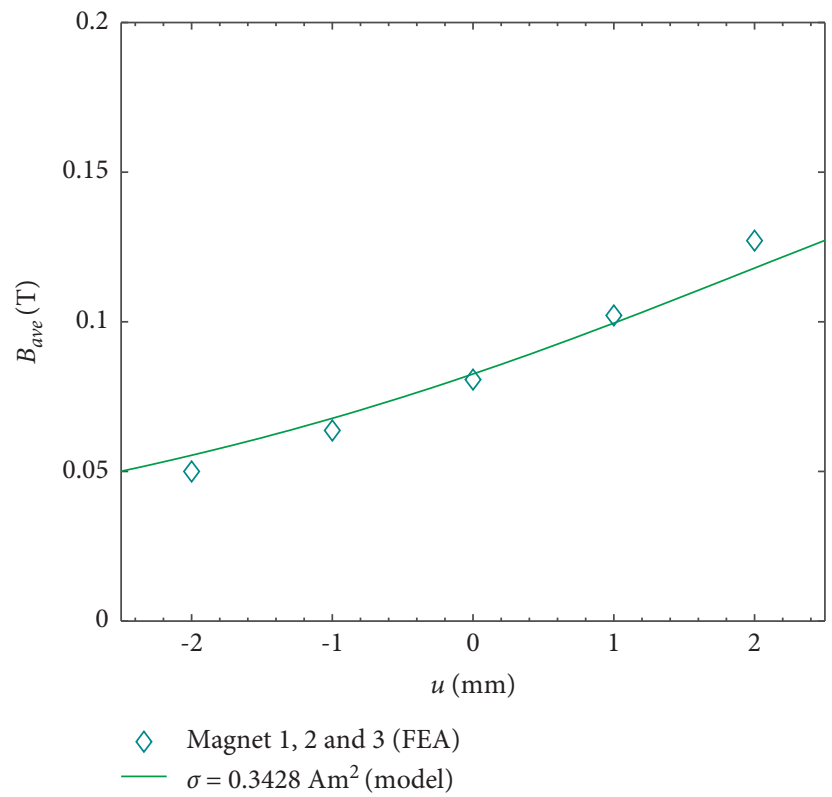

FIgURE 5: Average magnetic flux densities in the coil versus vibration displacement of the harvester.

the system in an excitation cycle. It can be seen from Figure 6(d) that there are multiple power peaks in a wide range of the frequency ratios. Define the power peaks from right to left, namely, the $1^{\text {st }}$ peak frequency is the dimensionless resonance frequency $\Omega_{1}$; it can be seen from Figure $6(\mathrm{~d})$ that the $i$ th dimensionless peak frequency $\Omega_{i}$ is almost equal to $\Omega_{1} / i(i=1,2,3, \ldots, 9)$. The maximal average power inputs occur at the nonresonant frequency $\Omega_{4}$ and not at the resonant frequency $\Omega_{1}$. It shows that the power inputs from a nonresonant excitation can be more efficient than those from a resonant excitation. It is noted that the input power is affected by both the driving force $F_{z}$ and the velocity $\dot{u}$ (see equation (19)). Due to the coupling between $F_{z}$ and $\dot{u}$, the driving force $F_{z}$ at each excitation is affected by the initial velocity of the beam (see Figures 6(a)-6(c)). It can be seen from Figures $6(\mathrm{a})$ and $6(\mathrm{~b})$ that when $\Omega=1$ and $1 / 4$, the effect of $\dot{u}$ on $F_{z}$ is not obvious. The difference is on that when $\Omega=1 / 4$, the direction of $\dot{u}$ is the same with the direction of $F_{z}$, which leads to a higher average power input than that at $\Omega=1$ (see Figure 6(d)). It can be seen from Figures 6(b) and 6(c) that a lower initial velocity at $\Omega=1 / 8$ leads to a lower driving force peak when compared with that at $\Omega=1 / 4$. Thus, the average power input at $\Omega=1 / 8$ is lower than that at $\Omega=1 / 4$ (see Figure $6(\mathrm{~d})$ ). It means that the optimal power input can occur at a dimensionless frequency with a higher vibration velocity $\dot{u}$ but with less effect on the input force $F_{\mathrm{z}}$. In addition, higher average input power is achieved when $\dot{u}$ and $F_{z}$ have the same direction. In this analysis, the optimal average input power occurs at $\Omega_{4}$.

Figures $7(a)$ and $7(b)$ show $3 \mathrm{D}$ plots of the average power inputs of the system with a series of $\Omega$ for various $\alpha$ and $\beta$, respectively. It can be seen from Figures 7 (a) and 7(b) that, at an arbitrary peak frequency, the average power inputs increase with a decrease in $\alpha$ and $\beta$, which implies that a larger dynamic response can occur at smaller $\alpha$ or at smaller $\beta$. It is noted that the proper selections of $\alpha$ and $\beta$ are necessary because the collision of magnet 2 with magnet 1 is easier to occur in a large displacement of the beam. Figure 8 shows the displacements and electromagnetic EMCS versus $\Omega$ at $\alpha=\beta=1$. It can be seen from Figure 8 that the range of the movement of the mass $m$ is from $-2 \mathrm{~mm}$ to $2 \mathrm{~mm}$ for the given parameter configuration. Since the absolute displacements of the mass $m$ are less than the initial gaps between two magnets $(|u|<2.5 \mathrm{~mm})$, the collision between magnets 2 and 1 does not occur. In Sections 5.2-5.4, the constant parameters $\alpha=\beta=1$ will be used for the analysis of the effect of the load resistance and the EMCSs on the system performance, respectively.

5.2. Effect of Dimensionless Load Resistance on the System Performance. Let the electromechanical parameters be given as $k_{p}^{2}=0.01$ and $\sigma=0.3428 \mathrm{Am}^{2}$. A parametric sweep simulation is performed in this analysis. Figures 9 (a) $-9(f)$ show $3 D$ plots of the maximal displacements, the maximal velocities, the average power inputs, the piezoelectric average power outputs, the electromagnetic average power outputs, and the power conversion efficiencies of the hybrid P-EMEH with a series of $\Omega$ and $r_{1}$ at $r_{2}=1$, respectively. It can be seen from Figures 9(a)9(e) that, at arbitrary $\Omega_{i}=\Omega_{1} / i(i=1,2,3, \ldots, 9)$, the displacements $u_{\max }$, the velocities $\dot{u}_{\max }$, the power inputs $\bar{P}_{\text {in }}$, and the electromagnetic power outputs $\bar{P}_{R 2}$ generate the valleys (see Figures 9(a)-9(c) and 9(e)). The piezoelectric power outputs $\bar{P}_{R 1}$ and the power conversion efficiencies $\eta$ generate the peaks (see Figure 9(d)). The occurrence of the peaks or the valleys is induced by the forward or backward actions of electromechanical energy conversion. When optimal $r_{1}$ is given to the piezoelectric transducer, the piezoelectric transducer can obtain the maximal power outputs. Under the backward action of the piezoelectric high power outputs, the vibrations of the harvester are relatively strongly suppressed, which leads to an obvious decrease in $\bar{P}_{\text {in }}$ and $\bar{P}_{R 2}$. The valleys occur at optimal $r_{1}$ 


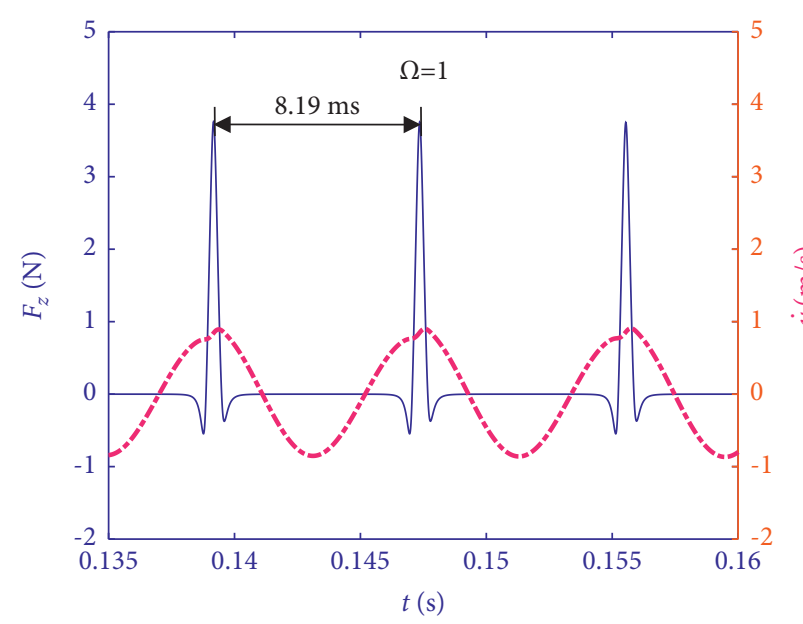

$\begin{array}{ll}- & F_{z} \\ -- & \dot{u}\end{array}$

(a)

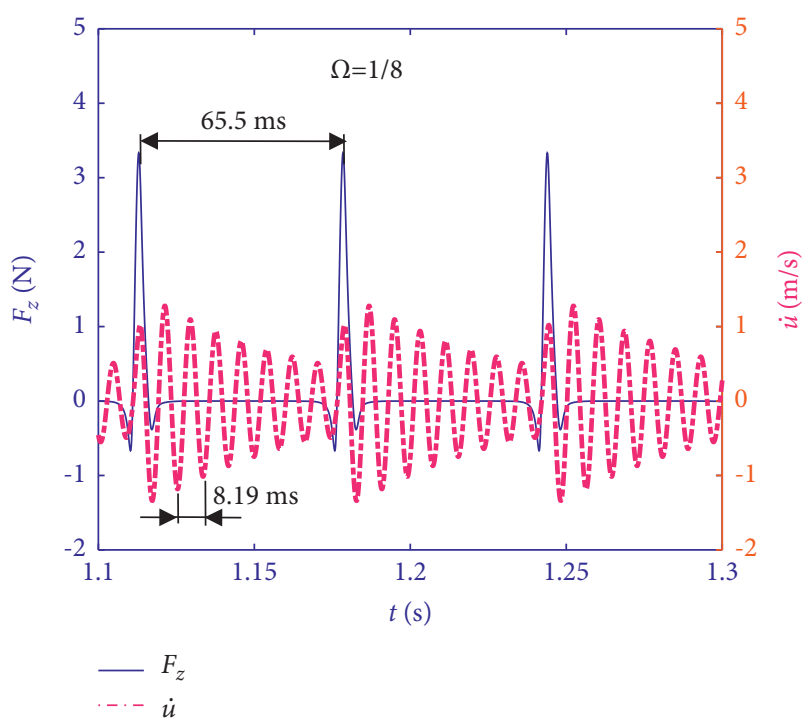

(c)

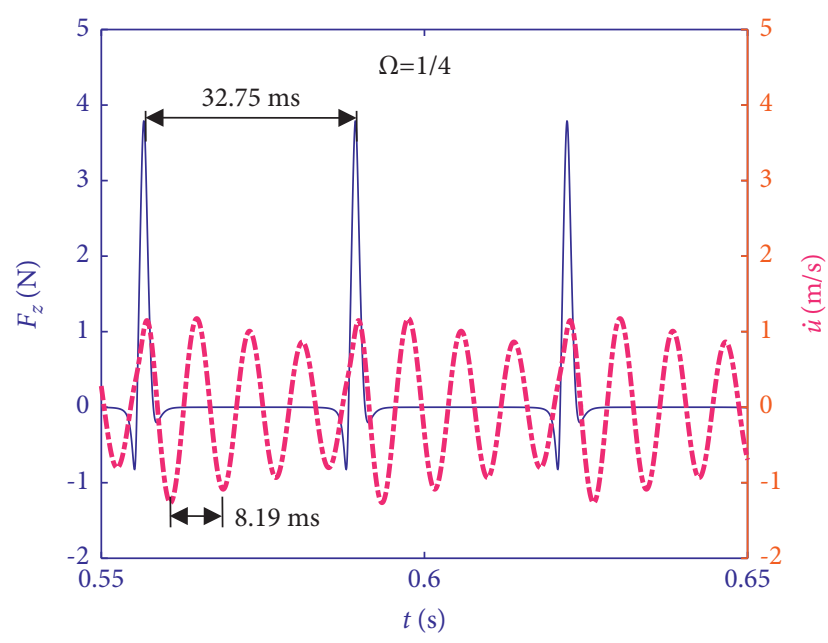

$-F_{z}$

$\cdot \cdot-\dot{u}$

(b)

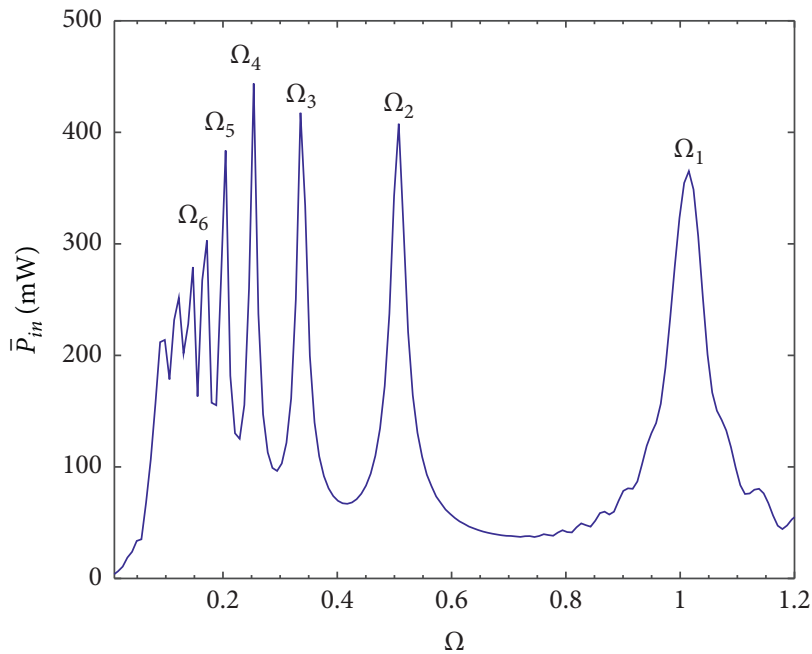

(d)

FiguRE 6: Time response of input force and velocity and frequency responses of average power inputs. (a) $\Omega=1$. (b) $\Omega=1 / 4$. (c) $\Omega=1 / 8$. (d) Average power inputs for various $\Omega . \alpha=\beta=1, r_{1}=r_{2}=1, k_{p}^{2}=0.01$, and $\sigma=0.3428 \mathrm{Am}^{2}$.

(see Figures 9(a)-9(c) and 9(e)). In Figure 9(f), the highest energy efficiency of the system appears at $\Omega_{4}$ and not at $\Omega_{1}$, which shows that the nonresonant energy harvesting can achieve higher power outputs than the resonant energy harvesting.

Figures $10(\mathrm{a})-10(\mathrm{f})$ show $3 \mathrm{D}$ plots of the maximal displacements, the maximal velocities, the average power inputs, the piezoelectric average power outputs, the electromagnetic average power outputs, and the efficiencies of the hybrid P-EMEH with a series of $\Omega$ and $r_{2}$ at $r_{1}=1$, respectively. It can be seen from Figures $10(\mathrm{a})-10(\mathrm{~d})$ that, at arbitrary $\Omega_{i}=\Omega_{1} / i$ $(i=1,2,3, \ldots, 9)$, with an increase in $r_{2}$, the displacements $u_{\max }$, the velocities $\dot{u}_{\max }$, the power inputs $\bar{P}_{\text {in }}$, and the piezoelectric power outputs $\bar{P}_{R 1}$ increase. The electromagnetic power outputs $\bar{P}_{R 2}$ and the power conversion efficiencies $\eta$ generate the peaks around $r_{1}=1$. The variation tendency can also be explained from the forward and backward actions of electromechanical energy conversion. When optimal $r_{2}$ is given to the electromagnetic element, the electromagnetic element can obtain the optimal power output (see Figure 10(e)). From the expression term of $\zeta_{e}$ given in equation (28), it can be seen that an increase in $r_{2}$ can lead to the decrease in $\zeta_{e}$. The total damping coefficient of the system decreases accordingly. Therefore, an increase in $r_{2}$ can induce the increase in $u$ and $\dot{u}$ (see Figures 10(a) and 10(b)). According to equations (19), (15), and (20), an increase in $\dot{u}$ and $u$ can induce the increase in $P_{\text {in }}$ and $\bar{P}_{R 1}$, respectively. Thus, $\bar{P}_{\text {in }}$ and $\bar{P}_{R 1}$ increase with the increase of $r_{2}$ (see Figures 10(c) and 10(d)). It can be seen from Figure $10(\mathrm{f})$ that the highest power conversion efficiency appears at $\Omega_{4}$. 


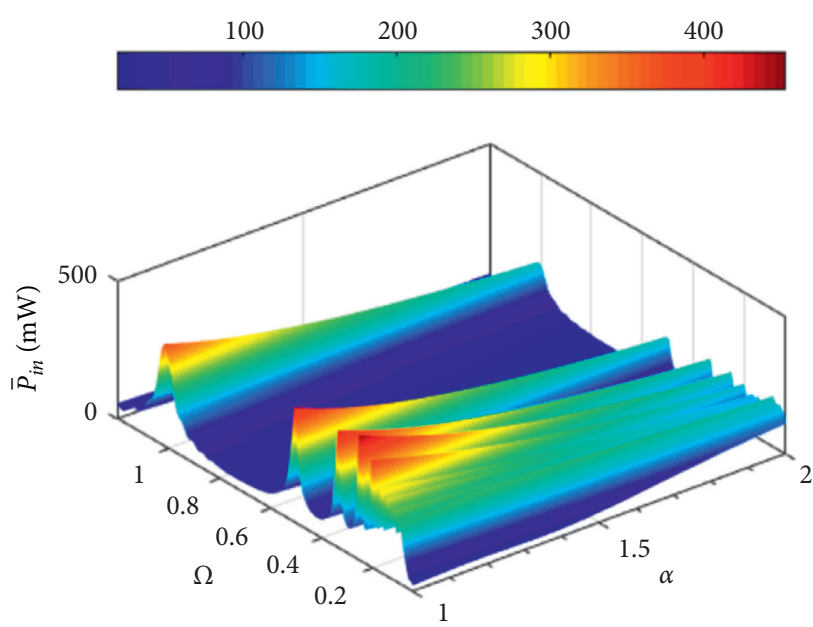

(a)

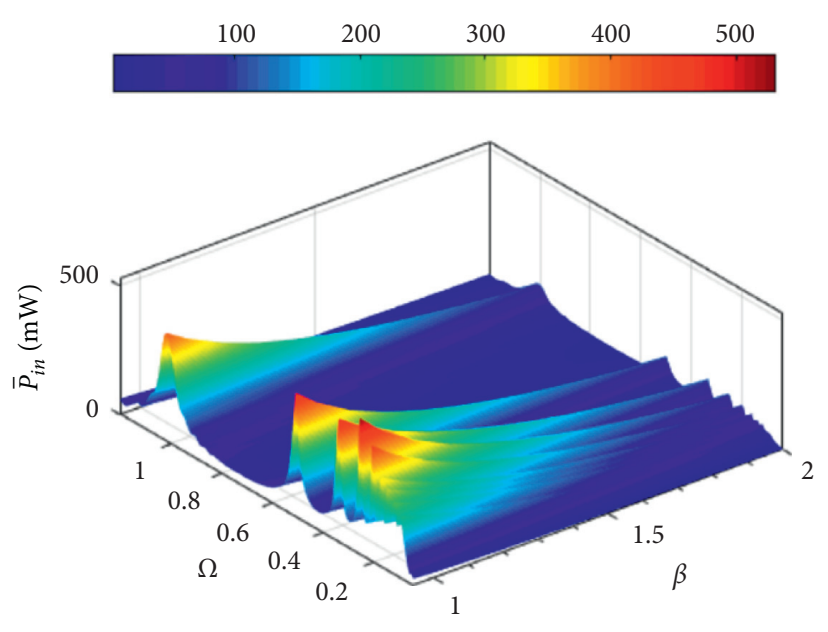

(b)

Figure 7: Average power inputs with a series of $\Omega$, $\alpha$, and $\beta$. (a) $\beta=1$. (b) $\alpha=1 . r_{1}=r_{2}=1, k_{p}^{2}=0.01$, and $\sigma=0.3428 \mathrm{Am}^{2}$.

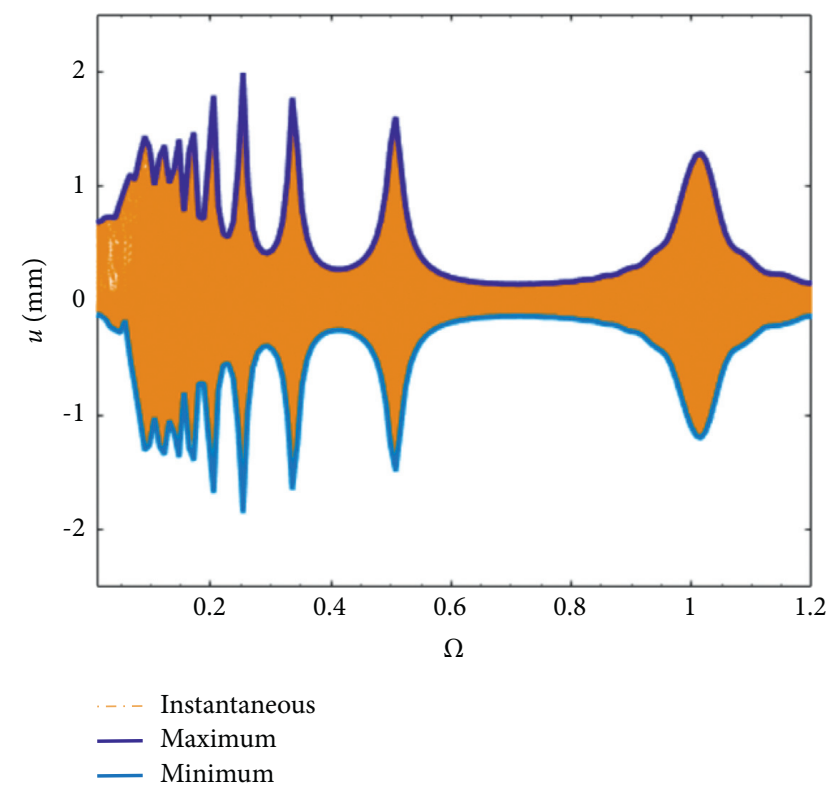

FIgURE 8: Displacement response of the mass $m$ versus $\Omega$ at $\alpha=\beta=1, r_{1}=r_{2}=1, k_{p}^{2}=0.01$, and $\sigma=0.3428 \mathrm{Am}^{2}$.

5.3. Effect of Electromechanical Coupling Strengths on the System Performance. Figures 11(a)-11(f) show 3D plots of the maximal displacements, the maximal velocities, the average power inputs, the piezoelectric average power outputs, the electromagnetic average power outputs, and the efficiencies of the hybrid P-EMEH with a series of $\Omega$ and $k_{p}^{2}$ at $r_{1}=r_{2}=1$ and $\sigma=0.3428 \mathrm{Am}^{2}$, respectively. It can be seen from Figures 11(a)-11(e) that, at arbitrary $\Omega_{i}=\Omega_{1} / i(i=1,2$, $3, \ldots, 9)$, the displacements $u_{\max }$, the velocities $\dot{u}_{\max }$, the power inputs $\bar{P}_{\text {in }}$, and the electromagnetic power outputs $\bar{P}_{R 2}$ decrease with an increase in $k_{p}^{2}$. The piezoelectric average power outputs $\bar{P}_{R 1}$ at each $\Omega_{i}(i=1,2, \ldots, 9)$ can increase until the saturation. The low-frequency saturation power is significantly higher than the high-frequency saturation power. Hence, the use of the hybrid P-EMEH with higher $k_{p}^{2}$ is advantageous for scavenging rotational energy at low frequencies. In Figure 11(f), the power conversion efficiencies of the system have the same tendency with the piezoelectric power outputs given in Figure 11(d). It shows that the piezoelectric energy conversions are dominant in the hybrid energy harvesting system due to the use of higher $k_{p}^{2}$.

To study the effect of the electromagnetic EMCS on the system performance, the average electromagnetic EMCSs in a rotational period are calculated. Figures 12 (a) and 12(b) show the instantaneous and average electromagnetic EMCSs versus $\Omega$ at $\sigma=0.3428 \mathrm{Am}^{2}$ and $\sigma=2.5313 \mathrm{Am}^{2}$, respectively. It can be seen from Figures 12(a) and 12(b) that, for an arbitrary given value of $\sigma$, the average electromagnetic EMCS can keep almost a constant value for various $\Omega$. A higher value of $\sigma$ can achieve higher $\bar{k}_{e}^{2}$. Figures 13(a)-13(f) show $3 \mathrm{D}$ plots of the maximal displacements, the maximal velocities, the average power inputs, the piezoelectric average power outputs, the electromagnetic average power outputs, and the efficiencies of the hybrid P-EMEH with a series of $\Omega$ and $\bar{k}_{e}^{2}$ at $r_{1}=r_{2}=1$ and $k_{p}^{2}=0.01$, respectively. It can be seen from Figures 13(a)-13(e) that the maximal displacements $u_{\max }$, the maximal velocities $\dot{u}_{\max }$, the average power inputs $\bar{P}_{\text {in }}$, and the piezoelectric average power outputs $\bar{P}_{R 1}$ decrease with the increase in $\bar{k}_{e}^{2}$. The average electromagnetic power outputs $\bar{P}_{R 2}$ can increase until the saturation at each $\Omega_{i}(i=1,2, \ldots, 9)$. Because low-frequency saturation power is significantly higher than high-frequency saturation power for the electromagnetic energy harvesting, it is favorable for the hybrid P-EMEH with higher $\bar{k}_{e}^{2}$ to harvest rotational energy at low frequencies. In Figure 13(f), the energy efficiencies of the system show the same tendency with the electromagnetic power outputs given in Figure 13(e). It shows that the electromagnetic energy conversions are dominant in the hybrid energy harvesting system due to the use of higher $\bar{k}_{e}^{2}$. 


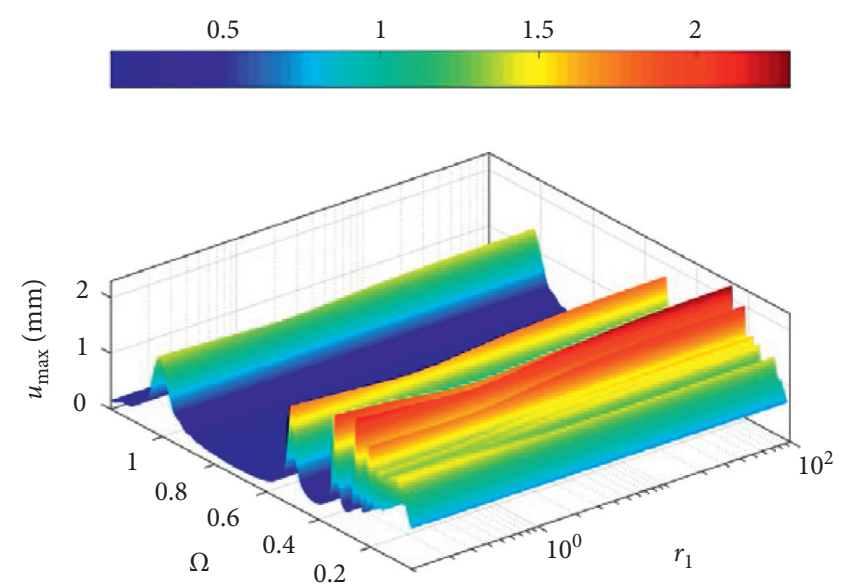

(a)

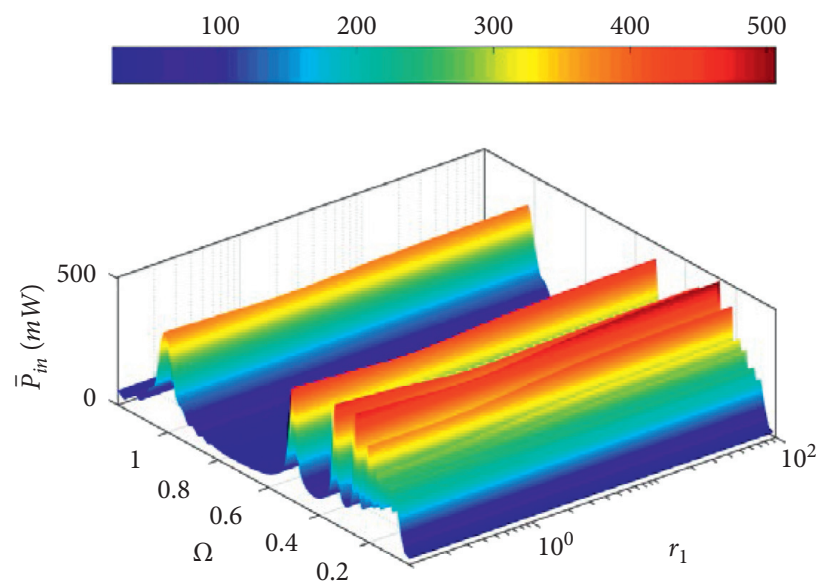

(c)
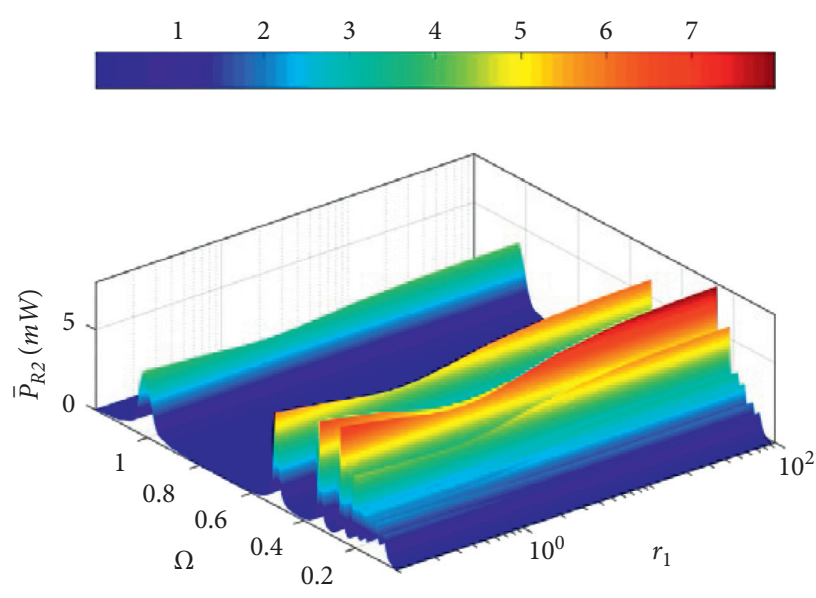

(e)

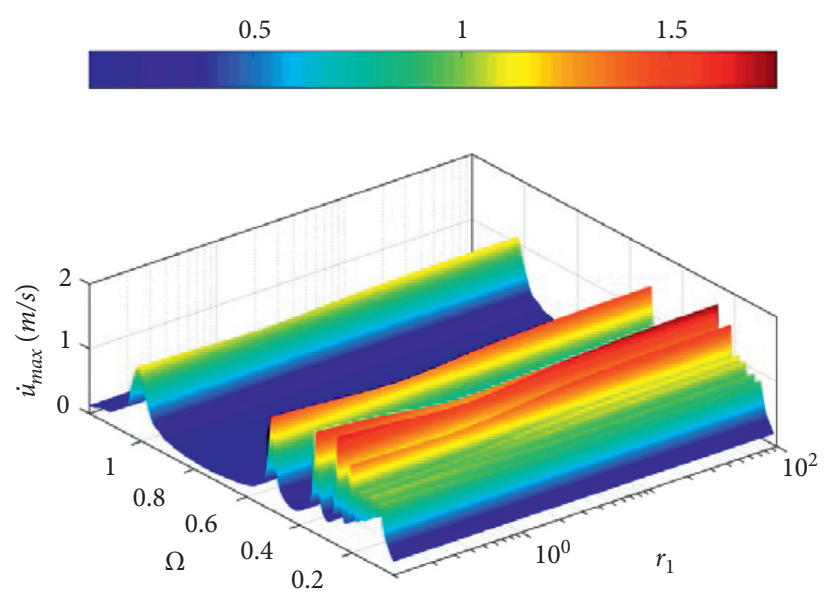

(b)

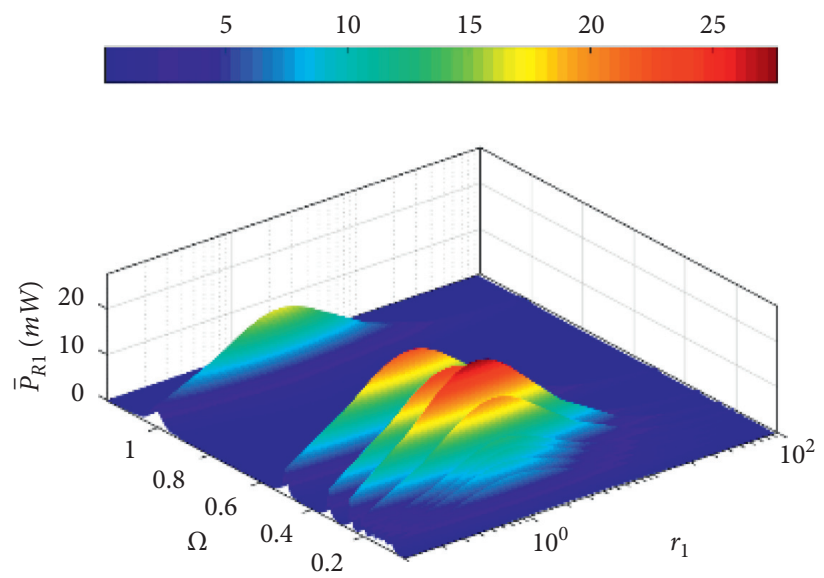

(d)
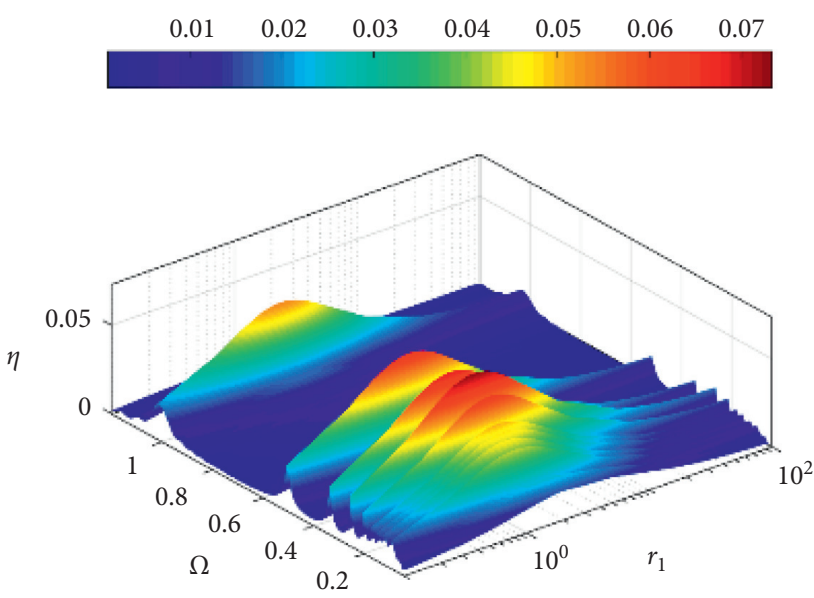

(f)

Figure 9: 3D plots for dynamic and electrical outputs with a series of $\Omega$ and $r_{1}$. (a) Maximal displacements. (b) Maximal velocities. (c) Average power inputs. (d) Piezoelectric average power outputs. (e) Electromagnetic average power outputs. (f) Power conversion efficiencies. $r_{2}=1$, $k_{p}^{2}=0.01$, and $\sigma=0.3428 \mathrm{Am}^{2}$. 


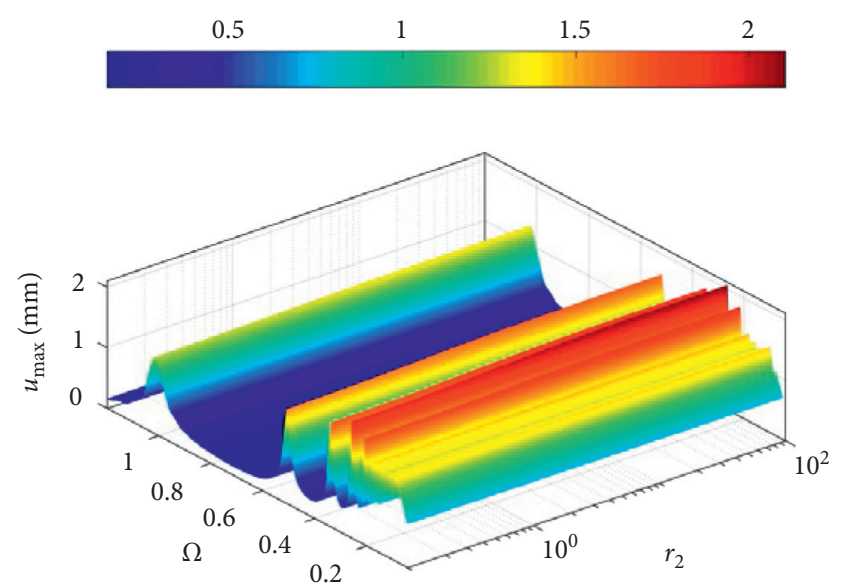

(a)

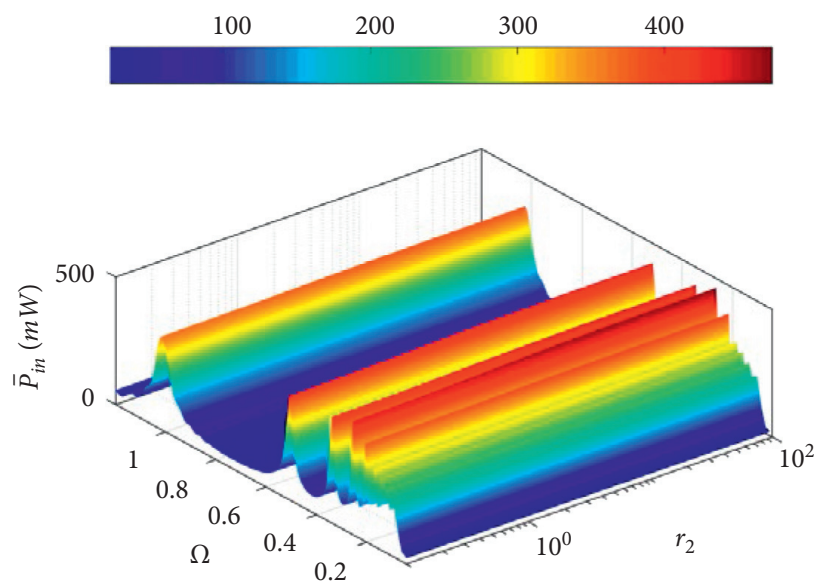

(c)
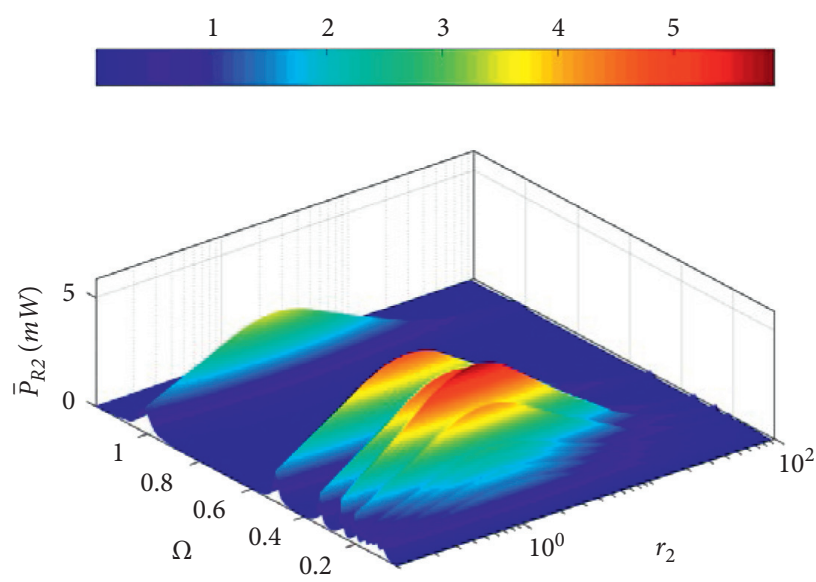

(e)

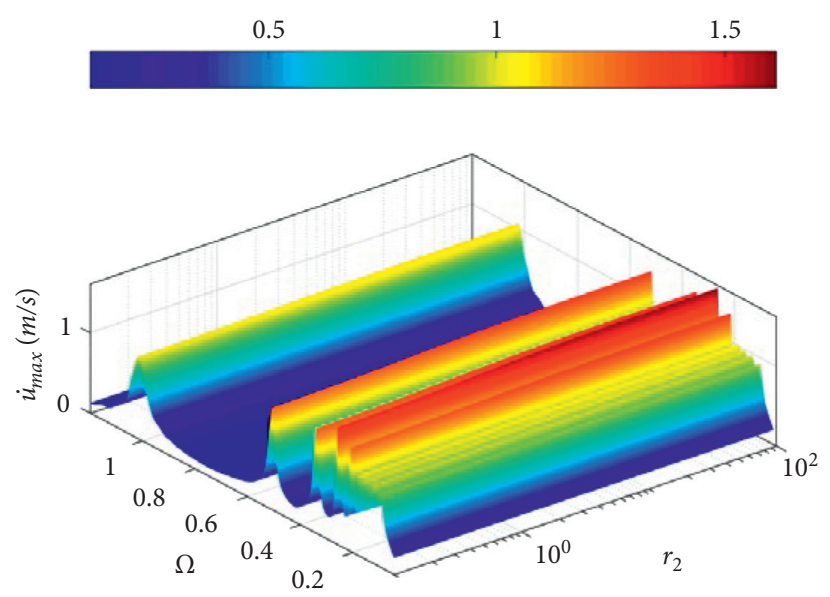

(b)

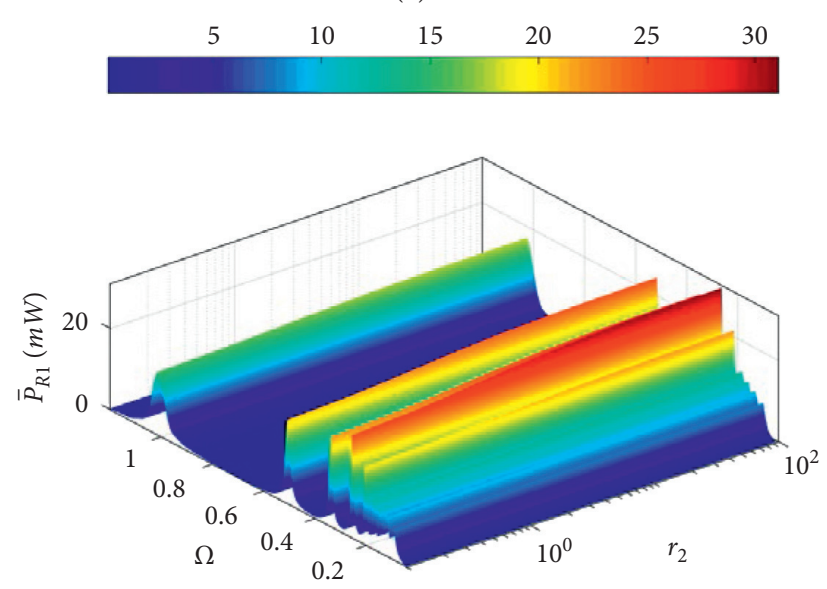

(d)
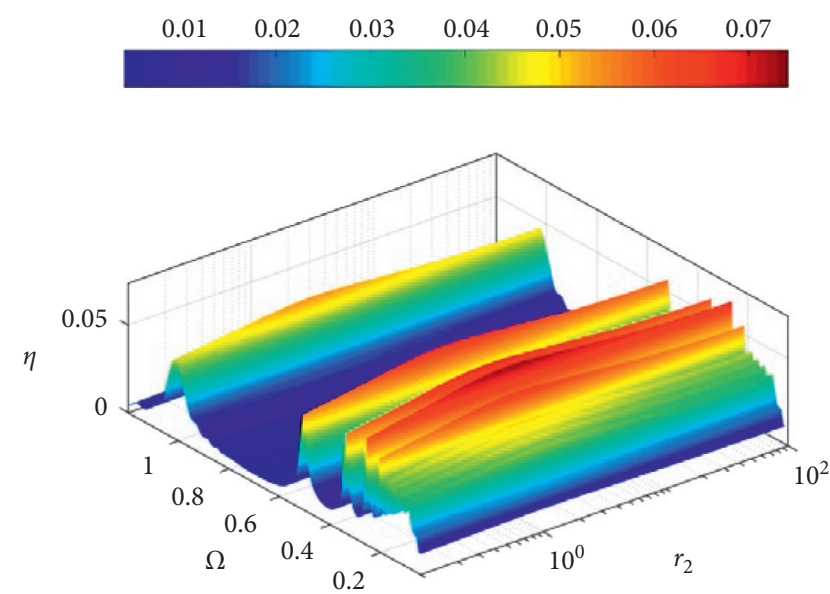

(f)

Figure 10: 3D plots for dynamic and electrical outputs with a series of $\Omega$ and $r_{2}$. (a) Maximal displacements. (b) Maximal velocities. (c) Average power inputs. (d) Piezoelectric average power outputs. (e) Electromagnetic average power outputs. (f) Power conversion efficiencies. $r_{1}=1$, $k_{p}^{2}=0.01$, and $\sigma=0.3428 \mathrm{Am}^{2}$. 


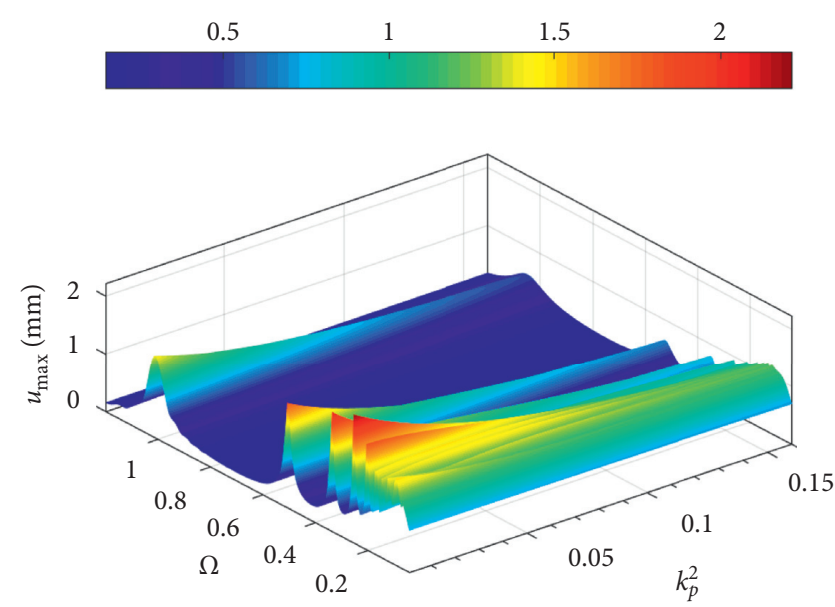

(a)
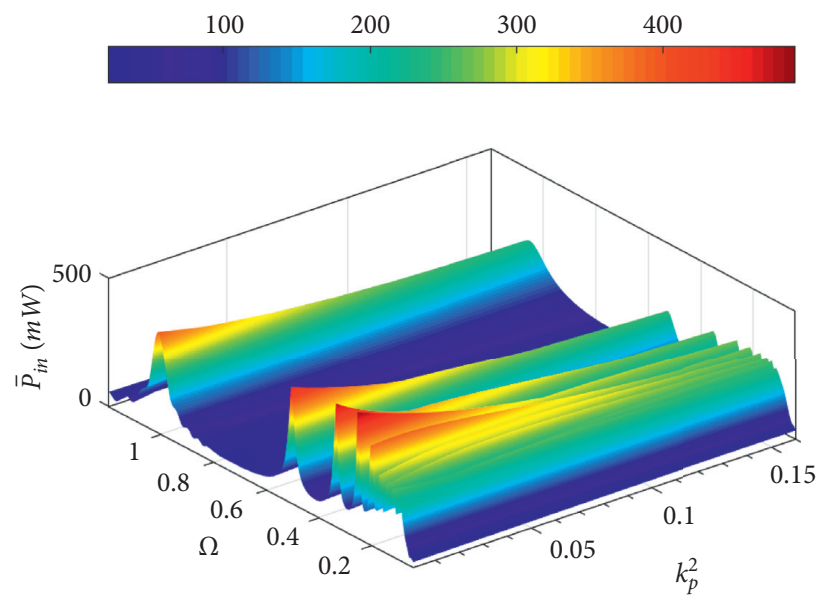

(c)

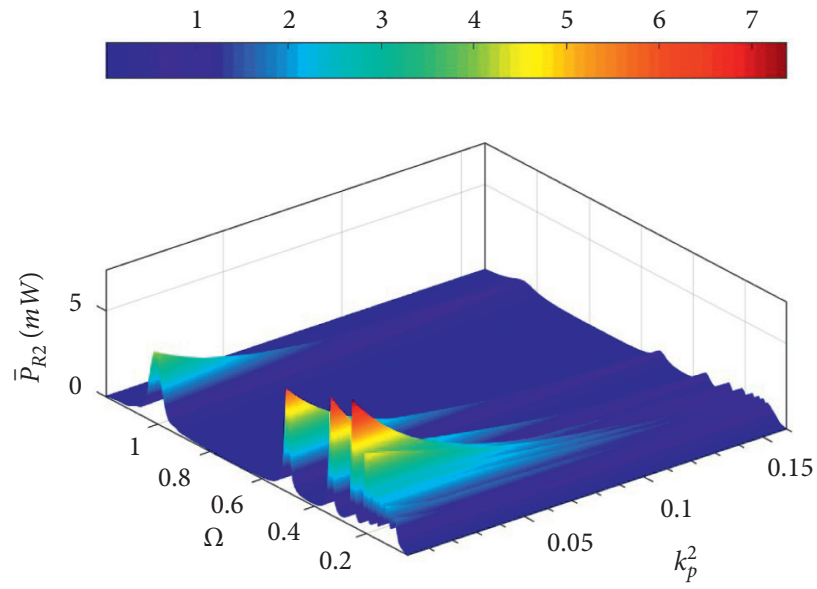

(e)

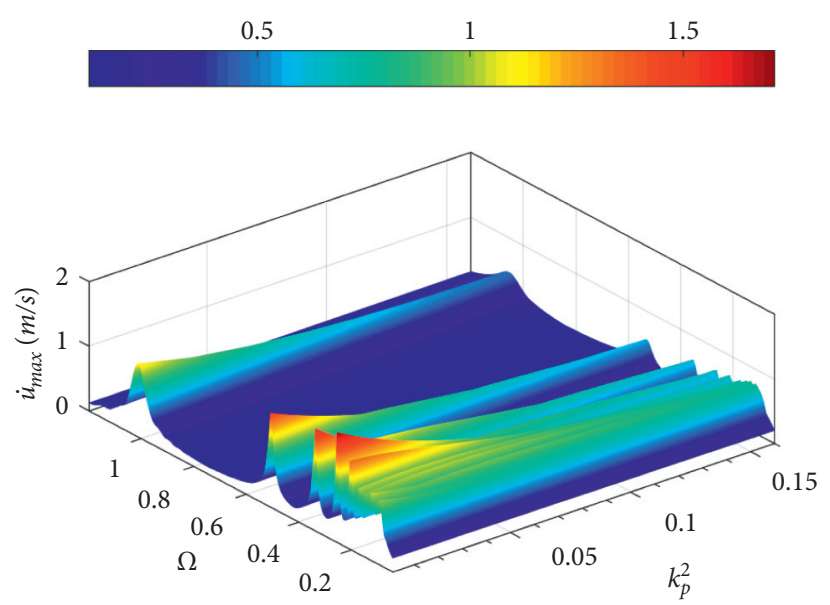

(b)

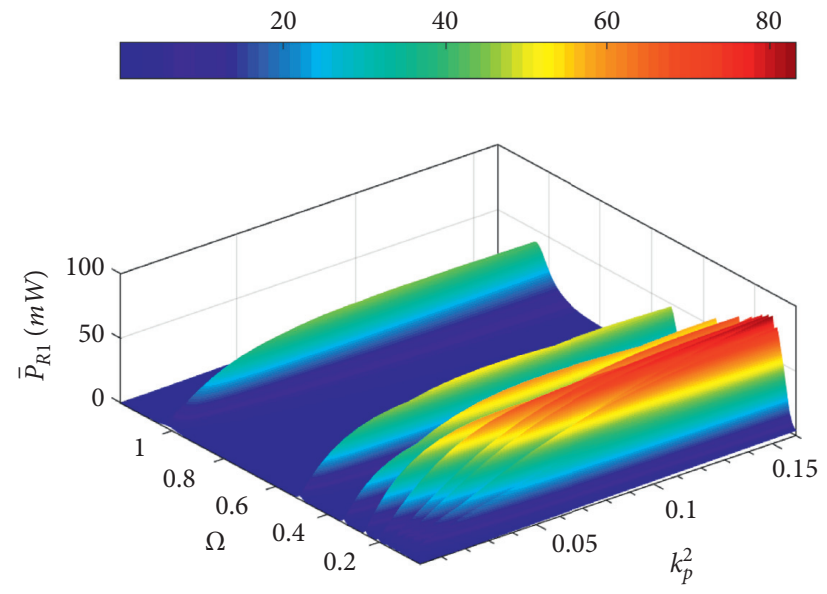

(d)

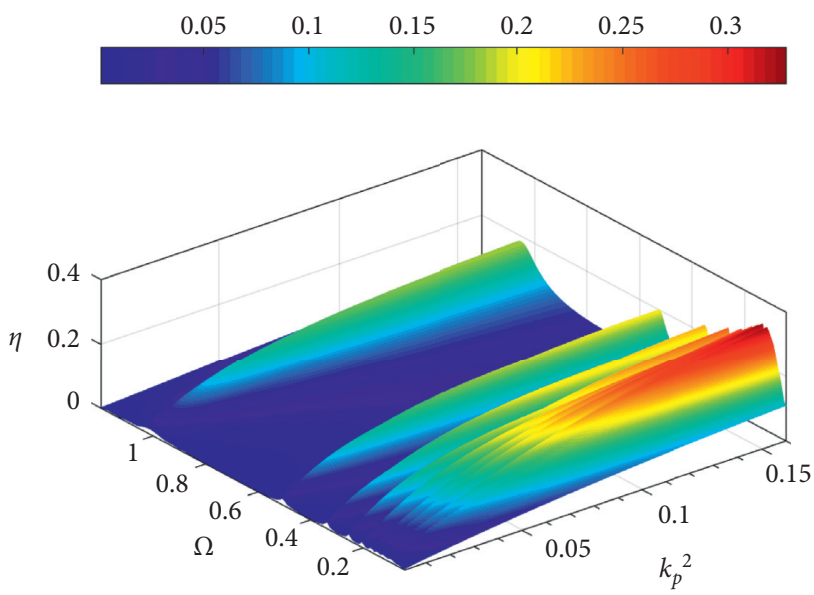

(f)

Figure 11: 3D plots for dynamic and electrical outputs with a series of $\Omega$ and $k_{p}^{2}$. (a) Maximal displacements. (b) Maximal velocities. (c) Average power inputs. (d) Piezoelectric average power outputs. (e) Electromagnetic average power outputs. (f) Power conversion efficiencies. $r_{1}=r_{2}=1$ and $\sigma=0.3428 \mathrm{Am}^{2}$. 


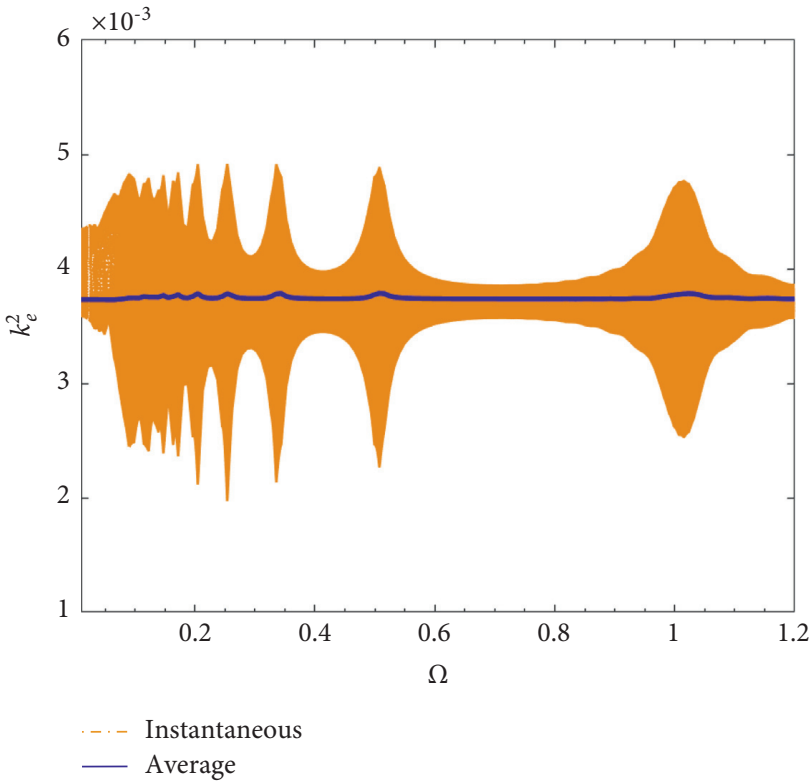

(a)

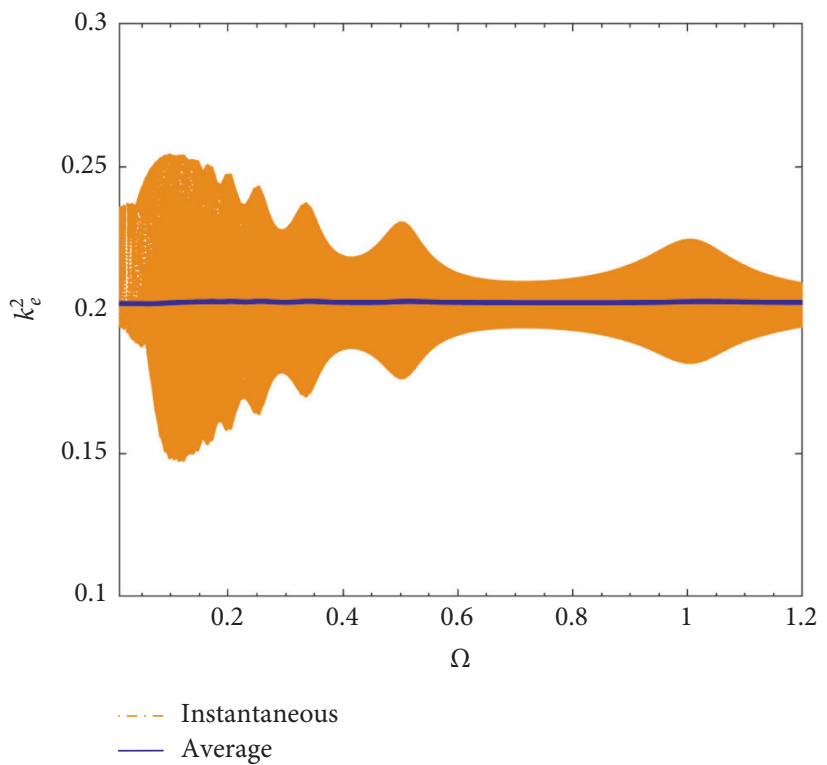

(b)

Figure 12: Instantaneous and average electromagnetic EMCSs versus $\Omega$ at $r_{1}=r_{1}=1$ and $k_{p}^{2}=0.01$. (a) $\sigma=0.3428 \mathrm{Am}^{2}$. (b) $\sigma=2.5313 \mathrm{Am}{ }^{2}$.

5.4. Power Comparison for the PEH, EMEH, and Hybrid $P$-EMEH at Weak and Strong Couplings. In this section, the power performance of the hybrid P-EMEH at weak and strong EMCS is compared with that from the single $\mathrm{PEH}$ and single EMEH. In order to distinguish strong coupling regions from weak ones, Shu and Lien [32] proposed using the parameter $\gamma_{1}=k_{p}^{2} / \zeta$ as the indicator of the EMCS of the PEH and defined a criterion of strong coupling, $\gamma_{1} \geq 4(\zeta+1)$, for a pure resistor circuit. They also referred to that the EMCS is very weak if $\gamma_{1} \ll 1$. Challa et al. [33] proposed using the parameter $\gamma_{2}=\theta_{e}^{2} /\left(R_{c} C\right)$ as the indicator of the EMCS of the EMEH and defined the weak coupling $\left(\gamma_{2} \ll 1\right)$ and the strong coupling $\left(\gamma_{2} \gg 1\right)$. Letting $k_{e}^{2}=\theta_{e}^{2} /\left(m \omega_{n} R_{c}\right)$ and $\zeta=C /(2 \sqrt{\mathrm{km}}), \gamma_{2}$ can be rewritten as $\gamma_{2}=k_{e}^{2} /(2 \zeta)$. Li et al. [34] proposed using the parameter $\gamma_{3}=\left(k_{p}^{2}+k_{e}^{2}\right) /(2 \zeta)$ as the indicator of the EMCS of the hybrid P-EMEH and defined the weak coupling $\left(\left(k_{p}^{2}+k_{e}^{2}\right) / \zeta \ll 2\right)$ and the strong coupling $\left(\left(k_{\theta}^{2}+k_{e}^{2}\right) / \zeta \gg 2\right)$. Based on these criteria above and letting $k_{e}^{2}=\bar{k}_{e}$ and supposing $\zeta=0.02$ in this work, the weak coupling is confined in the regions of $k_{p}^{2} \ll 0.02, \bar{k}_{e}^{2} \ll 0.04$, and $\left(k_{p}^{2}+\bar{k}_{e}^{2}\right) \ll 0.04$, respectively, for the $\mathrm{PEH}$, the EMEH, and the hybrid P-EMEH. The strong coupling is confined in the regions of $k_{p}^{2} \geq 0.0816, \bar{k}_{e}^{2} \gg 0.04$, and $\left(k_{p}^{2}+\bar{k}_{e}^{2}\right) \gg 0.04$, respectively, for the $\mathrm{PEH}$, the $\mathrm{EMEH}$, and the hybrid P-EMEH. Suppose $k_{p}^{2}=\bar{k}_{e}^{2}=0.002$ for the weak coupling and $k_{p}^{2}=\bar{k}_{e}^{2}=0.2$ for the strong coupling in this research.

Figures 14(a)-14(c) show the average power inputs, power outputs, and efficiencies for three different types of energy harvesters (PEH, EMEH, and hybrid P-EMEH) with optimal $r_{1}$ and $r_{2}$ at $k_{p}^{2}=\bar{k}_{e}^{2}=0.002$, respectively. In this study, optimal $r_{1}$ and $r_{2}$ are derived from the optimal average power outputs of the $\mathrm{PEH}$ and the EMEH, respectively, and they are used as the load resistance of the hybrid P-EMEH. It can be seen from Figures 14(a)-14(c) that there are close peak power inputs for the $\mathrm{PEH}$, the EMEH, and the hybrid $\mathrm{P}-\mathrm{EMEH}$. However, the hybrid P-EMEH has higher peak power outputs and efficiencies than the $\mathrm{PEH}$ and the EMEH. It shows that, under a weak coupling condition, higher $k_{p}^{2}$ can convert more mechanical energy into electricity with less effect on the dynamics of the system. Therefore, it is advantageous to use the hybrid P-EMEH to harvest the vibration energy under a weak electromechanical coupling condition.

Figures 15(a)-15(c) show the average power inputs, power outputs, and efficiencies for three different types of energy harvesters (PEH, EMEH, and hybrid P-EMEH) with optimal $r_{1}$ and $r_{2}$ at $k_{p}^{2}=\bar{k}_{e}^{2}=0.2$, respectively. It can be seen from Figures 15(a) and 15(b) that, for the PEH and the hybrid P-EMEH, there are obvious double peaks around each higher dimensionless frequency $\Omega_{i}(i=1,2,3,4)$. One is located at $\Omega_{i}(i=1,2,3$, and 4$)$, and another one is located at $\Omega_{i} \sqrt{1+k_{p}^{2}}(i=1,2,3$, and 4$)$. The occurrence of the double peaks can be reasonably explained from equation (28). In equation (28), when $r_{1} \longrightarrow 0$ (electrical short circuit), the dimensionless resonant frequency is $\Omega_{1}$. When $r_{1} \longrightarrow \infty$ (electrical open circuit), the dimensionless resonant frequency is $\Omega_{1} \sqrt{1+k_{p}^{2}}$. According to [35], the piezoelectric optimal power outputs can occur at both shortcircuit and open-circuit resonant frequencies. In this analysis, the double-peak power can be observed owing to higher $k_{p}^{2}$. Nonresonant energy harvesting has similar characteristics with resonant energy harvesting owing to the fact that the model frequency of the harvester is dominant in the vibration energy harvesting process, but with the decrease in $\Omega_{i}$ and $\Omega_{i} \sqrt{1+k_{p}^{2}}(i=2,3, \ldots, 9)$, the two nonresonant excitation frequencies become closer and 


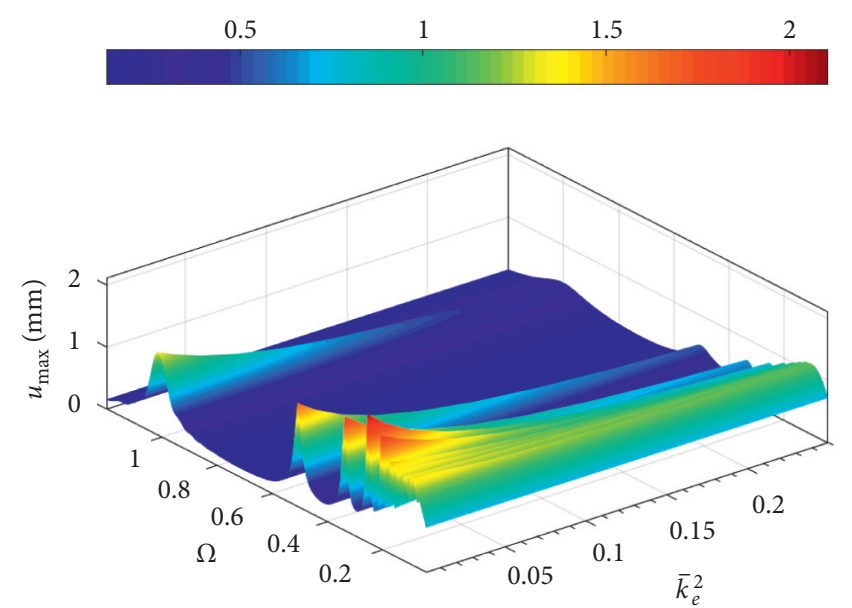

(a)

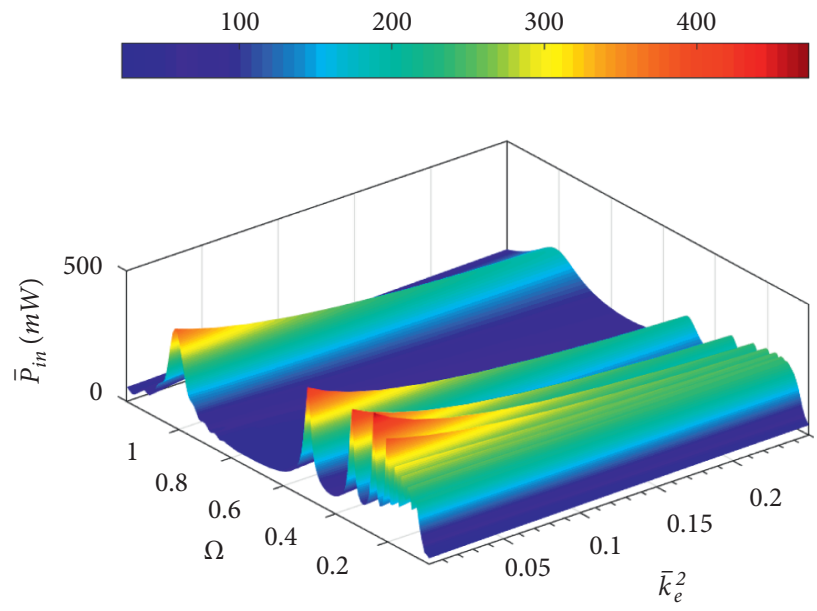

(c)

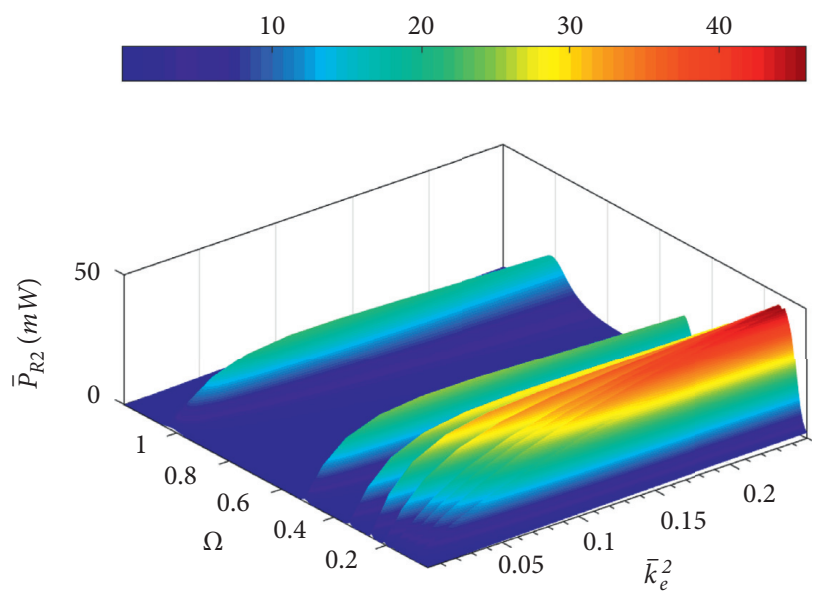

(e)

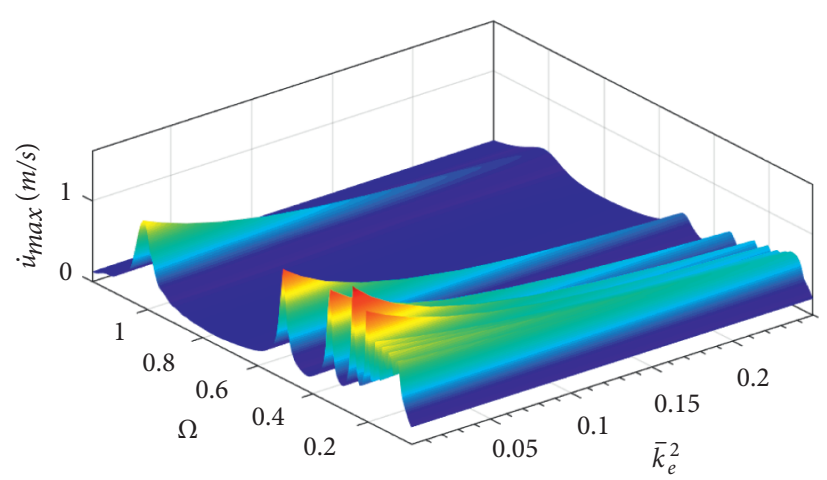

(b)
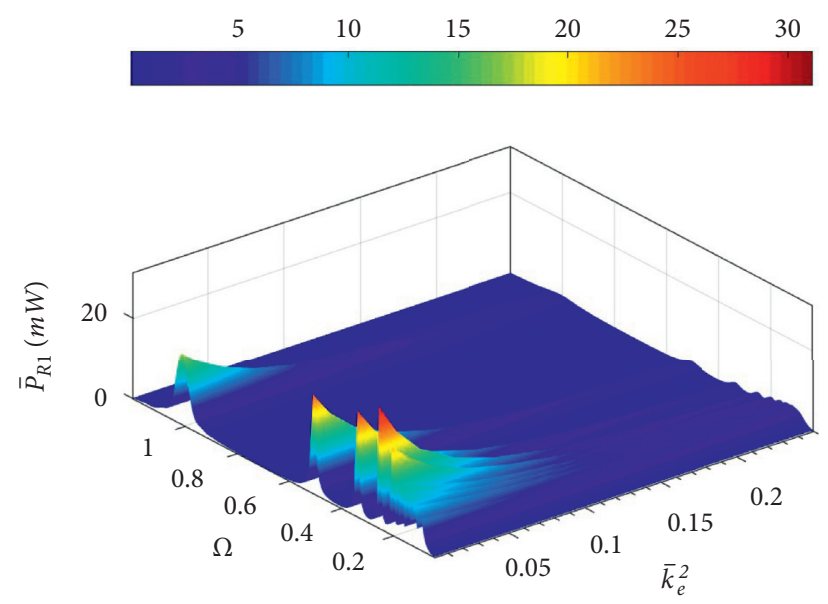

(d)

\begin{tabular}{llll}
0.05 & 0.1 & 0.15 & 0.2 \\
\hline
\end{tabular}

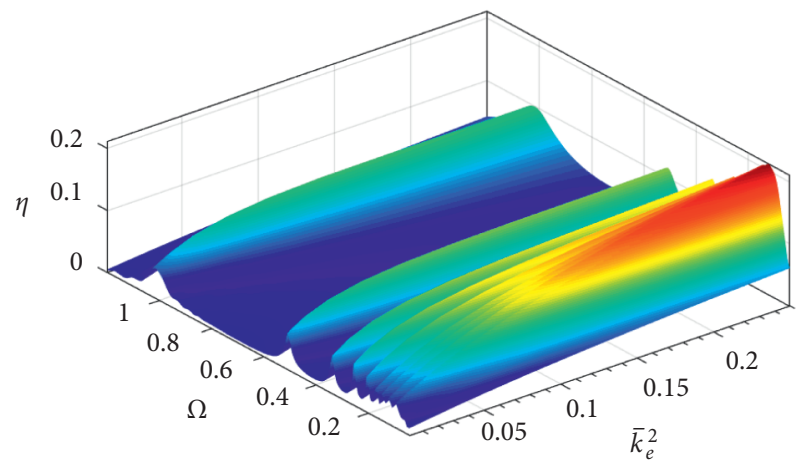

(f)

FIgURe 13: 3D plots for dynamic and electrical outputs with a series of $\Omega$ and $\bar{k}_{e}^{2}$. (a) Maximal displacements. (b) Maximal velocities. (c) Average power inputs. (d) Piezoelectric average power outputs. (e) Electromagnetic average power outputs. (f) Power conversion efficiencies. $r_{1}=r_{1}=1$ and $k_{p}^{2}=0.01$ 


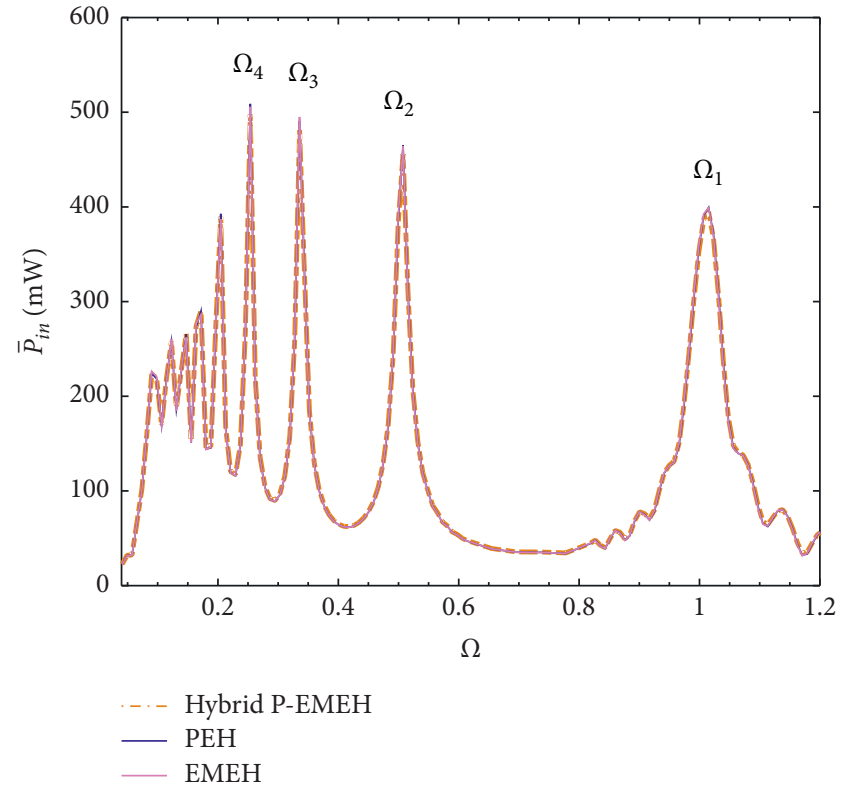

(a)

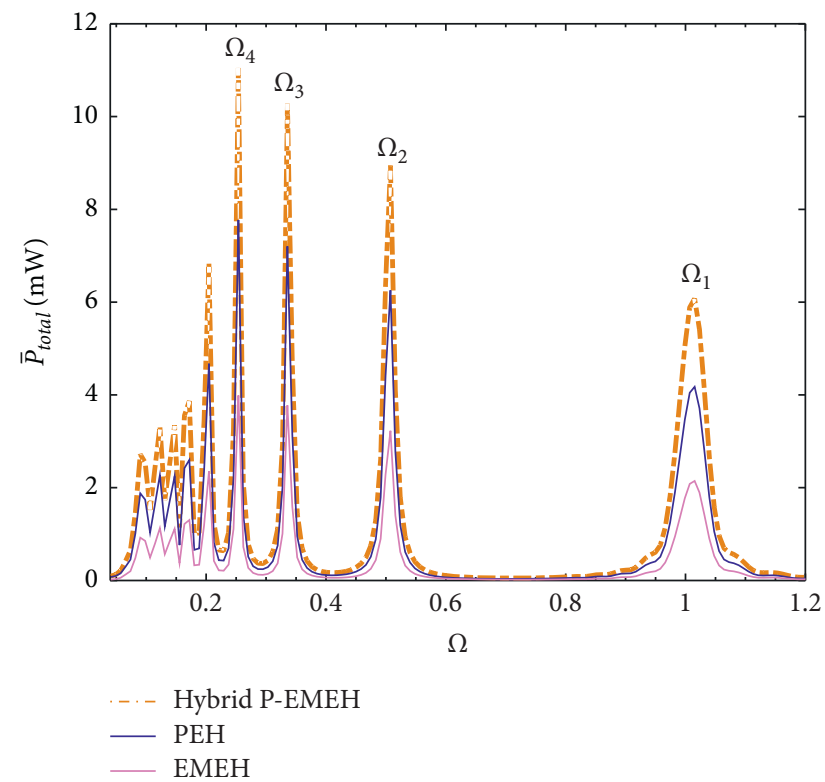

(b)

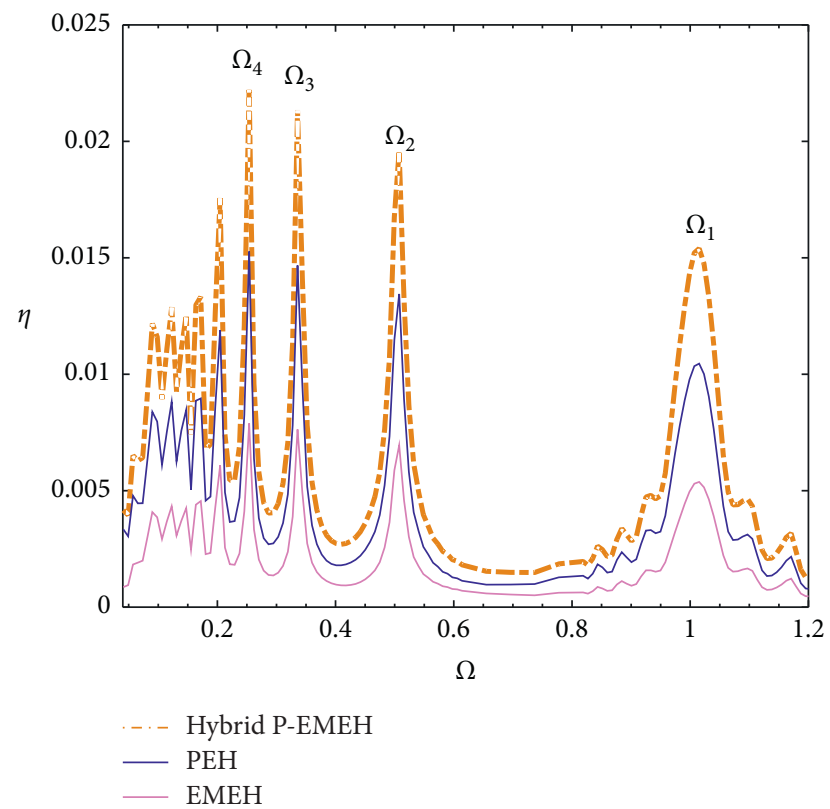

(c)

FIgURE 14: Power comparisons for the PEH, EMEH, and hybrid P-EMEH with optimal $r_{1}$ and $r_{2}$. (a) Average power inputs. (b) Total average power outputs. (c) Power conversion efficiencies. $k_{p}^{2}=\bar{k}_{e}^{2}=0.002$.

closer to each other until the frequency coincidence. Thus, there are single-peak power outputs at lower $\Omega_{i}$. The EMEH shows single-peak power characteristics owing to the fact that the electromagnetic EMCS is not relative to the resonance frequency of the harvester. It can be seen from Figure 15(b) that the PEH has wider power bandwidths than the hybrid P-EMEH at relatively high $\Omega_{i}(i=1,2,3,4)$, but it has close power outputs with the hybrid P-EMEH at relatively low $\Omega_{i}(i=5,6, \ldots, 9)$. The reason to this phenomenon can be explained by the power conversion efficiencies of the harvesters given in Figure 15(c). It can be seen from Figure 15(c) that, at higher $\Omega_{i}(i=1,2,3,4)$, the $\mathrm{PEH}$ and the hybrid P-EMEH have almost the same peak power efficiency, which means that power saturation occurs with given parameters $k_{p}^{2}=\bar{k}_{e}^{2}=0.2$. In this case, the same power outputs are theoretically possible for the hybrid EMEH and the PEH. However, owing to the singlepeak power characteristics of electromagnetic energy harvesting and its energy dissipation in the coil, the power outputs from the hybrid P-EMEH become lower than those from the $\mathrm{PEH}$ at higher $\Omega_{i}(i=1,2,3$, and 4) (see Figure $15(\mathrm{~b}))$. For lower $\Omega_{i}(i=5,6, \ldots, 9)$, it can be seen 


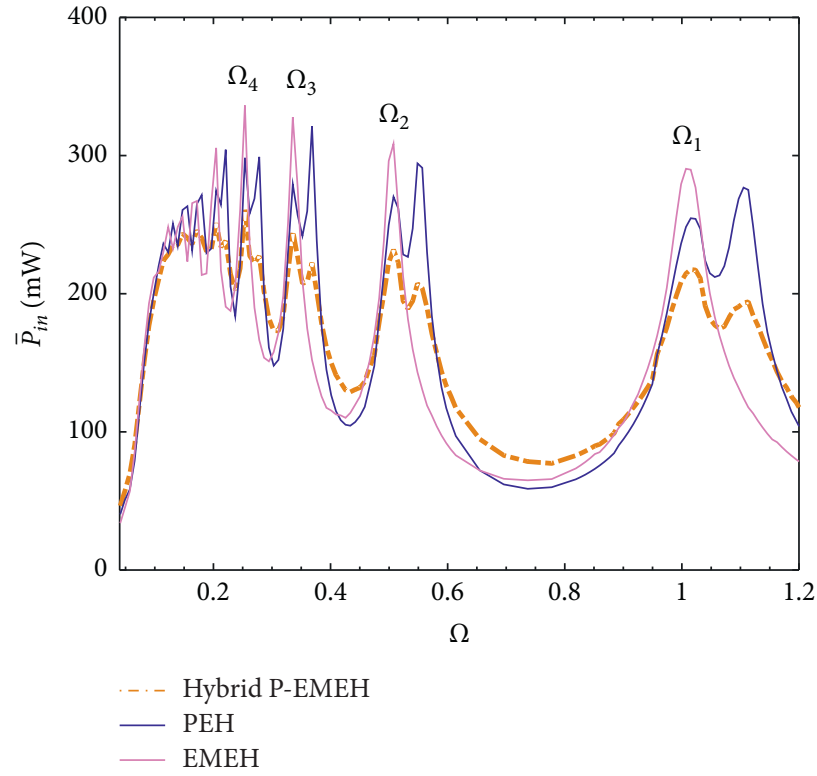

(a)

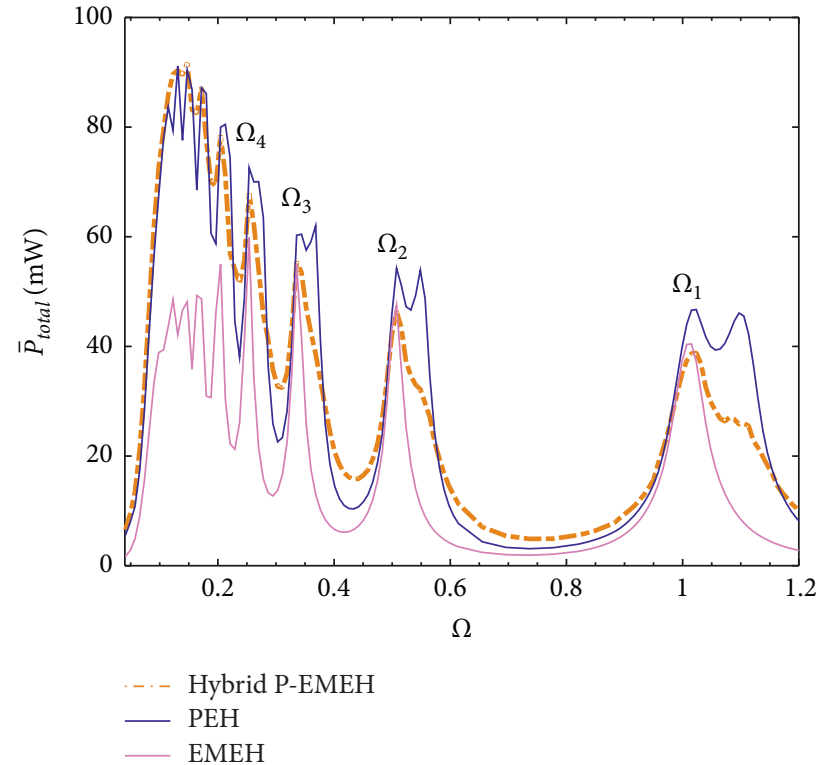

(b)

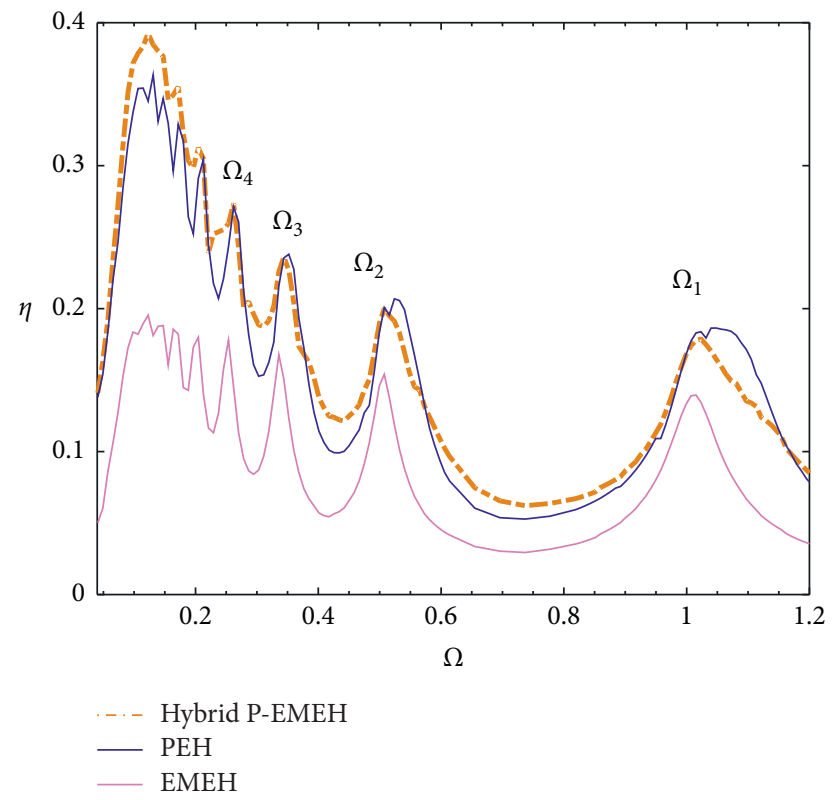

(c)

Figure 15: Power comparisons for the PEH, EMEH, and hybrid P-EMEH with optimal $r_{1}$ and $r_{2}$. (a) Average power inputs. (b) Total average power outputs. (c) Power conversion efficiencies. $k_{p}^{2}=\bar{k}_{e}^{2}=0.2$.

from Figure 15(c) that the peak power efficiency of the PEH is lower than that of the hybrid P-EMEH, which means that the piezoelectric power saturation cannot not be achieved with given parameter $k_{p}^{2}=0.2$ at lower $\Omega_{i}(i=5,6, \ldots, 9)$. Theoretically, the power outputs of the PEH should be lower than those of the hybrid P-EMEH. However, due to relatively less power loss in the piezoelectric element when compared with the electromagnetic element, the PEH can still provide almost the same power outputs with the hybrid $\mathrm{P}$-EMEH. The results show that it is more advantageous to use the single PEH to harvest the rotational vibration energy than the EMEH and hybrid P-EMEH under a strong electromechanical coupling condition. It can be seen from Figure 15(c) that the power conversion efficiencies of the three types of harvesters increase with a decrease in $\Omega_{i}$ $(i=1,2, \ldots, 9)$. A higher power conversion efficiency means that higher $k_{p}^{2}$ or $\bar{k}_{e}^{2}$ is required to achieve power saturation. It is the reason why the PEH with the same parameter $k_{p}^{2}=0.2$ can achieve power saturation at higher $\Omega_{i}(i=1,2$, $3,4)$, but not at lower $\Omega_{i}(i=5,6, \ldots, 9)$. It should be noted that, for the single EMEH, it has less advantage for magnetically plucked vibration energy harvesting at low frequencies due to relatively lower power outputs than those at middle and high frequencies (see Figure 15(b)). 
The significance of the analysis of the piezoelectric weak and strong coupling is that it provides a more comprehensive understanding on the power performance of the $\mathrm{PEH}$ in different coupling regimes. The results from weak coupling analysis show the advantages of higher power outputs of the hybrid P-EMEH when compared with the PEH and EMEH (see Figure 14(b)). The results from strong coupling analysis show the advantages of wider power bandwidths of the $\mathrm{PEH}$ at high dimensionless frequencies when compared with the hybrid P-EMEH and EMEH (see Figure 15(b)).

\section{Conclusions}

In this work, we investigate the dynamics and electrical characteristics of a hybrid P-EMEH driven by rotary magnetic plucking. The lumped-parameter model of the hybrid P-EMEH is established, and the model parameters including $\omega_{n}, k, m, k_{p}^{2}, J_{1}, J_{2}$, and $\sigma$ are determined based on the results from FEA. A parametric study is performed to investigate the effects of the dimensionless magnetic force parameters, excitation frequencies, load resistance, and EMCSs on the maximal displacements and velocities, average power inputs and outputs, and energy efficiencies of the system for indicating the performance of the hybrid P-EMEH. The optimal power outputs of the hybrid P-EMEH are analyzed and compared with those from the single PEH and the single EMEH under weak and strong coupling conditions. The results show that the hybrid P-EMEH has peak power inputs at a series of dimensionless frequencies $\Omega_{i}=\Omega_{1} / i(i=1,2,3, \ldots, 9)$, where $\Omega_{1}$ is the resonance frequency of the harvester. At each $\Omega_{i}$, the hybrid P-EMEH can obtain the improved average power inputs by reducing the rotational radii of the driving magnet and shortening the gaps between the two magnets. The structural vibrations can be strongly suppressed owing to the optimal piezoelectric power outputs, which can lead to the occurrence of valleys' power of the electromagnetic element. At weak coupling, the hybrid P-EMEH can achieve higher power outputs than the PEH and the EMEH. The use of the PEH strong coupling is more advantageous for energy harvesting due to wider operating bandwidths at high dimensionless frequencies when compared with those from the hybrid P-EMEH. The studies also show that the nonresonant rotational energy harvesting at some frequencies can be more efficient than the resonant rotational energy harvesting. The parametric study provides a fundamental understanding on the effect of load resistance and EMCSs on the performance of the hybrid P-EMEH driven by rotary magnetic plucking.

\section{Data Availability}

The data used to support the findings of this study are included within the article.

\section{Conflicts of Interest}

The authors declare that they have no conflicts of interest.

\section{Acknowledgments}

This research was funded by the Heilongjiang Provincial Natural Science Foundation for the Returned Overseas Chinese Scholars (Grant no. LC2017028), Fundamental Research Funds in Heilongjiang Provincial Universities of China (Grant no. 135209229), Innovative Research Program for Graduates of Qiqihar University (Grant no. YJSCX2020054), and Undergraduate Innovative Training Program of Qiqihar University (Grant no. 202010232608).

\section{References}

[1] H. Liu, H. Fu, L. Sun, C. Lee, and E. M. Yeatman, "Hybrid energy harvesting technology: from materials, structural design, system integration to applications," Renewable and Sustainable Energy Reviews, vol. 137, Article ID 110473, 2021.

[2] Z. Yang, S. Zhou, J. Zu, and D. Inman, "High-performance piezoelectric energy harvesters and their applications," Joule, vol. 2, no. 4, pp. 642-697, 2018.

[3] X. D. Xie, Q. Wang, and N. Wu, "Energy harvesting from transverse ocean waves by a piezoelectric plate," International Journal of Engineering Science, vol. 81, pp. 41-48, 2014.

[4] H. Kim, W. C. Tai, J. Parker, and L. Zuo, "Self-tuning stochastic resonance energy harvesting for rotating systems under modulated noise and its application to smart tires," Mechanical Systems and Signal Processing, vol. 122, pp. 769785, 2019.

[5] Y. Kuang and M. Zhu, "Characterisation of a knee-joint energy harvester powering a wireless communication sensing node," Smart Materials and Structures, vol. 25, no. 5, Article ID 055013, 2016.

[6] W.-H. Wu, K.-C. Kuo, Y.-H. Lin, and Y.-C. Tsai, "Noncontact magnetic cantilever-type piezoelectric energy harvester for rotational mechanism," Microelectronic Engineering, vol. 191, pp. 16-19, 2018.

[7] H. Liu, C. Hou, J. Lin et al., "A non-resonant rotational electromagnetic energy harvester for low-frequency and irregular human motion," Applied Physics Letters, vol. 113, no. 20, Article ID 203901, 2018.

[8] M. Perez, S. Boisseau, P. Gasnier, J. Willemin, M. Geisler, and J. L. Reboud, "A cm scale electret-based electrostatic wind turbine for low-speed energy harvesting applications," Smart Materials and Structures, vol. 25, no. 4, Article ID 045015, 2016.

[9] L. Lin, S. Wang, Y. Xie et al., "Segmentally structured disk triboelectric nanogenerator for harvesting rotational mechanical energy," Nano Letters, vol. 13, no. 6, pp. 2916-2923, 2013.

[10] F. Qian, W. Zhou, S. Kaluvan, H. Zhang, and L. Zuo, "Theoretical modeling and experimental validation of a torsional piezoelectric vibration energy harvesting system," Smart Materials and Structures, vol. 27, no. 4, Article ID 045018, 2018.

[11] F. Khameneifar, S. Arzanpour, and M. Moallem, "A piezoelectric energy harvester for rotary motion applications: design and experiments," IEEE, vol. 18, no. 5, pp. 1527-1534, 2013.

[12] L. Gu and C. Livermore, "Passive self-tuning energy harvester for extracting energy from rotational motion," Applied Physics Letters, vol. 97, p. R175, 2010. 
[13] M. Guan and W.-H. Liao, "Design and analysis of a piezoelectric energy harvester for rotational motion system," Energy Conversion and Management, vol. 111, pp. 239-244, 2016.

[14] M. Pozzi and M. Zhu, "Plucked piezoelectric bimorphs for knee-joint energy harvesting: modelling and experimental validation," Smart Materials and Structures, vol. 20, no. 5, Article ID 055007, 2011.

[15] M. A. Karami, J. R. Farmer, and D. J. Inman, "Parametrically excited nonlinear piezoelectric compact wind turbine," Renewable Energy, vol. 50, pp. 977-987, 2013.

[16] H. Fu and E. M. Yeatman, "A Methodology for low-speed broadband rotational energy harvesting using piezoelectric transduction and frequency up-conversion," Energy, vol. 125, pp. 152-161, 2017.

[17] Y. Zhang, A. Luo, Y. Wang, X. Dai, Y. Lu, and F. Wang, "Rotational electromagnetic energy harvester for human motion application at low frequency," Applied Physics Letters, vol. 116, no. 5, Article ID 053902, 2020.

[18] J. Kan, C. Fan, S. Wang, Z. Zhang, J. Wen, and L. Huang, "Study on a piezo-windmill for energy harvesting," Renewable Energy, vol. 97, pp. 210-217, 2016.

[19] T. Xue and S. Roundy, "On magnetic plucking configurations for frequency up-converting mechanical energy harvesters," Sensors and Actuators A: Physical, vol. 253, pp. 101-111, 2017.

[20] H. Fu and E. M. Yeatman, "Rotational energy harvesting using Bi-stability and frequency up-conversion for low-power sensing applications: theoretical modelling and experimental validation," Mechanical Systems and Signal Processing, vol. 125, pp. 229-244, 2019.

[21] G. Shi, J. Chen, Y. Peng et al., "A piezo-electromagnetic coupling multi-directional vibration energy harvester based on frequency up-conversion technique," Micromachines, vol. 11, no. 1, p. 80, 2020.

[22] Y. C. Shu, W. C. Wang, and Y. P. Chang, "Electrically rectified piezoelectric energy harvesting induced by rotary magnetic plucking," Smart Materials and Structures, vol. 27, no. 12, Article ID 125006, 2018.

[23] H. Allag and J. P. Yonnet, "3D analytical calculation of the forces exerted between two cuboidal magnets," IEEE Transactions on Magnetics, vol. 20, pp. 1962-1964, 1984.

[24] P. B. Landecker, D. D. Villani, and K. W. Yung, "An analytic solution for the torque between two magnetic dipoles," Magnetic and Electrical Separation, vol. 10, no. 1, pp. 29-33, 1999.

[25] B. A. M. Owens and B. P. Mann, "Linear and nonlinear electromagnetic coupling models in vibration-based energy harvesting," Journal of Sound and Vibration, vol. 331, no. 4, pp. 922-937, 2012.

[26] H.-y. Wang, L.-h. Tang, Y. Guo, X.-b. Shan, and T. Xie, "A 2DOF hybrid energy harvester based on combined piezoelectric and electromagnetic conversion mechanisms," Journal of Zhejiang University-Science, vol. 15, no. 9, pp. 711-722, 2014.

[27] T. Wacharasindhu and J. W. Kwon, "A micromachined energy harvester from a keyboard using combined electromagnetic and piezoelectric conversion," Journal of Micromechanics and Microengineering, vol. 18, no. 10, Article ID 104016, 2008.

[28] B. Yang, C. Lee, W. L. Kee, and S. P. Lim, "Hybrid energy harvester based on piezoelectric and electromagnetic mechanisms," Journal of Micro/Nanolithography, MEMS, and MOEMS, vol. 9, no. 2, Article ID 023002, 2010.

[29] N. E. duToit, B. L. Wardle, and S.-G. Kim, "Design considerations for mems-scale piezoelectric mechanical vibration energy harvesters," Integrated Ferroelectrics, vol. 71, no. 1, pp. 121-160, 2005.

[30] M. Kim, M. Hoegen, J. Dugundji, and B. L. Wardle, "Modeling and experimental verification of proof mass effects on vibration energy harvester performance," Smart Materials and Structures, vol. 19, no. 4, Article ID 045023, 2010.

[31] F. S. Tse, I. E. Morse, and R. T. Hinkle, Mechanical Vibrations Theory and Applications, Allyn \& Bacon, Boston, MA, USA, 2rd edition, 1978.

[32] Y. C. Shu and I. C. Lien, "Analysis of power output for piezoelectric energy harvesting systems," Smart Materials and Structures, vol. 15, no. 6, pp. 1499-1512, 2006.

[33] V. R. Challa, S. Cheng, and D. P. Arnold, "The role of coupling strength in the performance of electrodynamic vibrational energy harvesters," Smart Materials and Structures, vol. 22, no. 2, Article ID 025005, 2013.

[34] P. Li, S. Gao, H. Cai, and H. Wang, "Coupling effect analysis for hybrid piezoelectric and electromagnetic energy harvesting from random vibrations," International Journal of Precision Engineering and Manufacturing, vol. 15, no. 9, pp. 1915-1924, 2014.

[35] Y. Liao and H. A. Sodano, "Optimal parameters and power characteristics of piezoelectric energy harvesters with anRCcircuit," Smart Materials and Structures, vol. 18, no. 4, Article ID 045011, 2009. 